MATHEMATICS OF COMPUTATION

Volume 75 , Number 253, Pages 91-112

S 0025-5718(05)01787-4

Article electronically published on October 21, 2005

\title{
A SEMI-IMPLICIT MONOTONE DIFFERENCE SCHEME FOR AN INITIAL-BOUNDARY VALUE PROBLEM OF A STRONGLY DEGENERATE PARABOLIC EQUATION MODELING SEDIMENTATION-CONSOLIDATION PROCESSES
}

\author{
RAIMUND BÜRGER, ANÍBAL CORONEL, AND MAURICIO SEPÚLVEDA
}

\begin{abstract}
We prove the convergence of a semi-implicit monotone finite difference scheme approximating an initial-boundary value problem for a spatially one-dimensional quasilinear strongly degenerate parabolic equation, which is supplied with two different inhomogeneous flux-type boundary conditions. This problem arises in the modeling of the sedimentation-consolidation process. We formulate the definition of entropy solution of the model in the sense of Kružkov and prove convergence of the scheme to the unique $B V$ entropy solution of the problem, up to satisfaction of one of the boundary conditions.
\end{abstract}

\section{INTRODUCTION}

In this paper we prove convergence of a semi-implicit difference scheme for an initial-boundary value problem for a scalar strongly degenerate parabolic equation. This problem arises from a model of sedimentation-consolidation processes of particulate suspensions [1, 2, 9. However, under slight modifications, it can also be understood as a spatially finite model of two-phase flow in porous media [16] or traffic flow with driver reaction [5, 26].

The initial-boundary value problem (IBVP) is given by

$$
\begin{aligned}
\phi_{t}+f(\phi, t)_{x} & =A(\phi)_{x x}, \quad(x, t) \in Q_{T}:=I \times \mathcal{T}, \\
\phi(x, 0) & =\phi_{0}(x), \quad x \in I:=(0,1), \\
\left(f(\phi, t)-A(\phi)_{x}\right)(1, t) & =\Psi(t), \quad t \in \mathcal{T}:=(0, T), \\
\left(b(\phi)-A(\phi)_{x}\right)(0, t) & =0, \quad t \in \mathcal{T},
\end{aligned}
$$

where $t$ is time, $x$ is the spatial coordinate, $T$ is the final time, $\phi$ is the unknown function, $f(\phi, t)=q(t) \phi+b(\phi)$ is the total flux, where $q(t)$ is a control function

Received by the editor May 18, 2004 and, in revised form, January 18, 2005.

2000 Mathematics Subject Classification. Primary 35L65, 35R05, 65M06, 76T20.

Key words and phrases. Degenerate parabolic equation, monotone scheme, upwind difference scheme, boundary conditions, entropy solution.

We acknowledge support by FONDECYT projects 1030718 and 1050728, Fondap in Applied Mathematics, the German Acadamic Exchange Service (DAAD) and CONICYT (Chile) through project Alechile/DAAD/CONICYT 2003154, and the Sonderforschungsbereich 404 at the University of Stuttgart.

(C)2005 American Mathematical Society Reverts to public domain 28 years from publication 
and $b(\phi)$ is a material-dependent flux density function, and

$$
A(\phi):=\int_{0}^{\phi} a(s) d s, \quad a(\phi) \geqslant 0,
$$

where the diffusion function $a(\phi)$ is allowed to vanish on intervals of positive length.

In the sedimentation-consolidation model, the coordinate $x$ increases vertically, and $\phi$ is the sought solids volume fraction. The IBVP (1.1) describes a onedimensional ideal continuous thickener for the settling of industrial suspensions; see 1, 2, 9] for details. The flux boundary condition (1.1c) models that at the top boundary $x=1$, a feed source is located, through which fresh suspension is fed into the unit at the feed rate $\Psi(t)$ (see 4 for details). At the bottom $(x=0)$, the total solids flux $f(\phi, t)-A(\phi)_{x}$ is reduced to its convective part $q(t) \phi(0, t)$, which leads to the boundary condition (1.1d). A subcase included here is that of batch settling of a suspension in a closed column, which corresponds to setting $q \equiv 0$.

The basic assumptions on $q(t), b(\phi)$ and $A(\phi)$ and on the initial and boundary data arising from the sedimentation-consolidation model are the following. The function $q(t) \leqslant 0$ is the volume-averaged velocity of the suspension, which can be controlled, while $b(\phi)$ is a continuous, piecewise smooth function satisfying

$$
b(\phi)=0 \text { for } \phi \leqslant 0 \text { and } \phi \geqslant \phi_{\max }, \quad b(\phi)<0 \text { for } \phi \in\left(0, \phi_{\max }\right),
$$

where $\phi_{\max } \in(0,1]$ is the maximum concentration value. The function $b(\phi)$ models the concentration-dependent hindrance of the settling of a solid particle due to the presence of other particles. The degenerating diffusion function $a(\phi)$ models the sediment compressibility, and is assumed to satisfy

$$
a(\phi)=0 \text { for } \phi \leqslant \phi_{\mathrm{c}} \text { and } \phi \geqslant \phi_{\max }, \quad a(\phi)>0 \text { for } \phi_{\mathrm{c}}<\phi<\phi_{\max },
$$

where $\phi_{\mathrm{c}} \in\left[0, \phi_{\max }\right]$ is a critical concentration at which the solid particles get into contact with each other. The functions $a(\phi)$ and $b(\phi)$ reflect the specific material properties of the suspension. Since (1.1a) reduces to a first-order conservation law on the interval of positive length $\left[0, \phi_{\mathrm{c}}\right]$, this equation is called strongly degenerate. Finally, the initial function $\phi_{0}(x)$ is piecewise continuous with $0 \leq \phi_{0}(x) \leqslant \phi_{\max }$, while the feed flux $\Psi(t) \leqslant 0$ must be larger than the minimum of $f(\cdot ; t)$.

We mention that an analysis of the IBVP (1.1) is given in 2]. Since solutions of (1.1a) are discontinuous in general, they need to be defined as weak solutions along with an entropy condition to select the physically relevant weak solution. In 2] the existence of $B V$ entropy weak solutions to (1.1) in the sense of Kružkov [25] and Vol'pert and Hudjaev [28, 29] is shown via the vanishing viscosity method, while their uniqueness is shown by a technique introduced by Carrillo 10. On the other hand, Evje and Karlsen [19] show that explicit monotone finite difference schemes, which were first introduced by Crandall and Majda [12 for conservation laws, converge to $B V$ entropy solutions for initial-value problems of equation (1.1a) (in the slightly simpler case that the flux does not depend on $t$ ). These results are extended to implicit schemes in [18], and to several space dimensions in [22]. The extension of these schemes to initial-boundary value problems with flux-type boundary conditions is utilized for the simulation of sedimentation-consolidation processes defined by (1.1) in a number of papers including [1, 3, 8, 21, to which we refer for numerical examples illustrating the scheme and the model.

In [4, the analyses of [2] and [19] are summarized, and a detailed error study of the monotone scheme presented herein (as well as of a MUSCL extrapolation 
to formal second-order spatial accuracy) is presented. However, convergence of the scheme is not proved in 4]. This paper supplies this convergence analysis, which in part relies on [19, 22] but includes some new proofs required by the boundary conditions. We analyze a semi-implicit scheme. It should be clear that similar arguments can be applied to the explicit and fully implicit variants of the scheme.

Convergence of monotone schemes to an entropy solution has also been proved for conservation laws and strongly degenerate convection-diffusion equations with discontinuous flux [6, 7, 8, 23, 24. Such equations arise, for example, if the sedimentation-consolidation model is extended to so-called clarifier-thickener units. In addition, the present analysis is important for the justification of the numerical parameter identification scheme advanced in 11.

The remainder of this paper is organized as follows. In Section 2, we recall the definition of an entropy solution of (1.1) and the characterization of the traces for this kind of degenerate parabolic equation, and we describe the semi-implicit numerical scheme. In Section 3 we prove that the semi-implicit scheme is well defined, which means that the nonlinear equations defining the scheme always have a unique solution. This property is established by proving that the scheme is monotone and $L^{\infty}$ stable. (The solution to the nonlinear equations can be computed, for example, by the Newton-Raphson method.) In Section 4, we derive $B V$ estimates for the numerical solution and Lipschitz continuity estimates of the discrete integrated diffusion function. Finally, in Section [5, we show that the scheme satisfies a cell entropy inequality which permits us to prove that the discrete solutions converge to a limit that satisfies the entropy condition. We also show that the limit satisfies the initial condition and one of the boundary conditions. Combining the results of Sections 4 and 5. we obtain that the scheme converges to a solution that satisfies all ingredients of the definition of entropy weak solutions except for the boundary condition at $x=0$. Available numerical results, however, also indicate that this boundary condition is properly approximated, and for the special case $q=0$, the analysis of Section 5 implies that the limit does satisfy the boundary condition at $x=0$, and therefore is the unique entropy weak solution of (1.1).

\section{Preliminaries}

\subsection{Definition of an entropy solution.}

Definition 2.1. A measurable function $\phi=\phi(x, t)$ is an entropy solution of the initial-boundary value problem (1.1) if the following conditions are satisfied:

(S1) $\phi \in L^{\infty}\left(Q_{T}\right) \cap B V\left(Q_{T}\right)$.

(S2) $A(\phi) \in C^{1,1 / 2}\left(Q_{T}\right)$.

(S3) For all test functions $\varphi \in C_{0}^{\infty}\left(Q_{T}\right), \varphi \geqslant 0$, and any $k \in \mathbb{R}$, the following entropy inequality holds:

$$
\iint_{Q_{T}}\left\{|\phi-k| \varphi_{t}+\operatorname{sgn}(\phi-k)\left[f(\phi, t)-f(k, t)-A(\phi)_{x}\right] \varphi_{x}\right\} d x d t \geqslant 0 .
$$

(S4) The boundary condition at $x=0$ is satisfied in the following sense:

$$
\gamma_{0}\left(b(\phi)-A(\phi)_{x}\right)=0 \quad \text { for almost all } t \in \mathcal{T},
$$

where $\gamma_{0} v$ denotes the trace of $v$ with respect to $x \downarrow 0$.

(S5) The boundary condition at $x=1$ is satisfied in the following sense:

$$
\gamma_{1}\left(f(\phi, t)-A(\phi)_{x}\right)=\Psi(t) \text { for almost all } t \in \mathcal{T},
$$


where $\gamma_{1} v$ denotes the trace of $v$ with respect to $x \uparrow 1$.

(S6) The initial condition is satisfied in the following sense:

$$
\lim _{t \rightarrow 0} \phi(x, t)=\phi_{0}(x) \quad \text { for almost all } x \in I .
$$

The traces $\gamma_{0} \phi$ and $\gamma_{1} \phi$ are well defined by the result given in [30.

The existence, uniqueness and stability of the entropy solutions for (1.1) was proved in [2] under the following hypotheses:

(H1) The function $b$ is continuous, piecewise differentiable with $\left\|b^{\prime}\right\|_{\infty} \leq \infty$, and satisfies (1.2).

(H2) The function $q$ is locally Lipschitz continuous such that $q(t) \leq 0$ for almost all $t \in \mathcal{T}, \operatorname{TV}_{\mathcal{T}}(q)<\infty$, and $\operatorname{TV}_{\mathcal{T}}\left(q^{\prime}\right)<\infty$.

(H3) The function $a$ is bounded, piecewise continuous with $\operatorname{supp} a \subset \operatorname{supp} b$, and satisfies (1.3).

(H4) The function $\Psi$ satisfies $\operatorname{TV}_{\mathcal{T}}(\Psi)<\infty$ and

$$
\min _{\phi \in\left[0, \phi_{\max }\right]} f(\phi, t) \leq \Psi(t) \leq 0, \quad \Psi(t) \geq f\left(\phi_{\max }, t\right), \quad t \in \overline{\mathcal{T}} .
$$

$\left(\mathrm{H} 5^{\prime}\right)$ The initial datum $\phi_{0}$ belongs to the set

$$
\begin{aligned}
\mathscr{B}^{\prime}:=\{ & \phi \in B V(I): \phi(x) \in\left[0, \phi_{\max }\right] \forall x \in \bar{I} \\
& \left.\wedge \exists M_{0}>0: \operatorname{TV}_{I}\left(\partial_{x} A_{\epsilon}(\phi)\right)<M_{0} \text { uniformly in } \epsilon\right\},
\end{aligned}
$$

where $A_{\epsilon}(\phi)$ is a smooth, positive regularization of $A(\phi)$. This condition is in particular satisfied if $\phi_{0}$ is a constant.

Condition $\left(\mathrm{H} 5^{\prime}\right)$, stated in [2], refers to the viscous approximation of (1.1), and is required for a uniform estimate of the spatial variation of the time derivative of the solutions of the regularized, strictly parabolic problem. We here need to replace $\left(\mathrm{H} 5^{\prime}\right)$ by a condition that involves the spatial discretization. To this end, let $J \in \mathbb{N}$ denote the number of space steps, $\Delta x:=1 / J, x_{-1 / 2}:=0, x_{J+1 / 2}:=1$, $x_{j+1 / 2}:=(j+1 / 2) \Delta x$ for $j=0, \ldots, J-1, I_{j}:=\left[x_{j-1 / 2}, x_{j+1 / 2}\right)$, and

$$
\phi_{j}^{0}:=\frac{1}{\Delta x} \int_{I_{j}} \phi_{0}(x) d x, \quad j=0, \ldots, J .
$$

The new condition can be stated as follows.

(H5) The initial datum belongs to the set

$$
\begin{aligned}
\mathscr{B}:=\{ & \phi_{0} \in B V(I): \phi_{0}(x) \in\left[0, \phi_{\max }\right] \forall x \in \bar{I} \wedge \exists M_{0}, M_{1}>0: \\
& \left|A\left(\phi_{1}^{0}\right)-A\left(\phi_{0}^{0}\right)\right| \leqslant M_{0} \Delta x \\
& \left.\wedge \sum_{j=1}^{J-1}\left|A\left(\phi_{j+1}^{0}\right)-2 A\left(\phi_{j}^{0}\right)+A\left(\phi_{j-1}^{0}\right)\right| \leqslant M_{1} \Delta x \text { uniformly in } \Delta x\right\} .
\end{aligned}
$$

This condition is satisfied, for example, if $\phi_{0}$ is constant. Moreover, in [2] the existence of an entropy solution was proved under the condition that either $\Psi=0$ or there exist positive constants $\xi$ and $M_{g}$ such that $\xi a(\phi)-\left(q(t)+b^{\prime}(\phi)\right) \geq M_{g}$. This condition, which is due to $\mathrm{Wu}$ [27, is not required in this work. Instead, we impose the following condition. 
(H6) Either (a) $q(t)=0$ and $\Psi(t)=0$ for all $t \in \mathcal{T}$, or (b) $q(t)<0$ for all $t \in \mathcal{T}$, and there exists a piecewise continuous functions $\varphi(t)$ such that $u=\varphi(t)$ is the unique solution of $f(u, t)=\Psi(t)$ for all $t \in \mathcal{T}$, i.e., the function $\varphi(t)$ denotes the time-dependent intersection of the functions $f_{1}(u ; t):=f(u, t)$ and $f_{2}(u ; t)=\Psi(t)$, and we assume that there exists a constant $C_{1}$ such that $\operatorname{TV}_{\mathcal{T}}(\varphi) \leqslant C_{1}$.

The distinction between cases (a) and (b) in (H6) is made for simplicity of the argument only; it will become clear that our analysis remains valid for more general situations, e.g., when $q(t)=\Psi(t)=0$ on a finite number of intervals $\mathcal{T}_{1}, \ldots, \mathcal{T}_{\mathcal{N}} \subset \mathcal{T}$ and $\varphi(t)$ is defined as in case (b) on the remaining segments $\mathcal{T} \backslash\left(\mathcal{T}_{1} \cup \cdots \cup \mathcal{T}_{\mathcal{N}}\right)$.

2.2. The difference scheme. Let $N \in \mathbb{N}$ be the number of time steps, $\Delta t:=T / N$, and $I^{n}:=\left[t^{n}, t^{n+1}\right)$, where $t^{n}:=n \Delta t$ for $n=0, \ldots, N$. We denote by $\phi_{j}^{n}$ the numerical solution at $\left(x_{j}, t_{n}\right)$, and assume that the values for $n=0$ are given by (2.5). The semi-implicit scheme is defined by an "interior" formula, which is consistent to the governing equation (1.1a), and two "boundary" schemes that are produced by inserting the discrete versions of the boundary conditions (1.1d) and (1.1c) into the interior formula, respectively, where $n=0, \ldots, N-1$ :

$$
\begin{aligned}
& \phi_{0}^{n+1}=\phi_{0}^{n}-\lambda g_{1 / 2}^{n}+\mu d_{1 / 2}^{n+1}+\lambda q\left(t^{n}\right) \phi_{0}^{n} \\
& \phi_{j}^{n+1}=\phi_{j}^{n}-\lambda\left(g_{j+1 / 2}^{n}-g_{j-1 / 2}^{n}\right)+\mu\left(d_{j+1 / 2}^{n+1}-d_{j-1 / 2}^{n+1}\right), \quad j=1, \ldots, J-1, \\
& \phi_{J}^{n+1}=\phi_{J}^{n}+\lambda g_{J-1 / 2}^{n}-\mu d_{J-1 / 2}^{n+1}-\lambda \Psi\left(t^{n}\right) .
\end{aligned}
$$

Here we let $\lambda:=\Delta t / \Delta x, \mu:=\Delta t / \Delta x^{2}$, and

$$
d_{j+1 / 2}^{n}:=A\left(\phi_{j+1}^{n}\right)-A\left(\phi_{j}^{n}\right), \quad g_{j+1 / 2}^{n}:=g\left(\phi_{j}^{n}, \phi_{j+1}^{n}, t^{n}\right),
$$

where $g(u, v, t)$ is the numerical flux. We assume that $g: \mathbb{R}^{3} \rightarrow \mathbb{R}$ is a locally Lipschitz continuous function such that $g$ restricted to $\left[0, \phi_{\max }\right]^{2} \times[0, T]$ is nondecreasing in its first argument and nonincreasing in its second, and $g$ is consistent with $f$, i.e., $g(\phi, \phi, t)=f(\phi, t)$ for all $(\phi, t) \in\left[0, \phi_{\max }\right] \times[0, T]$. Moreover, we assume that the linear portion $q(t) \phi$ of the total flux $f(\phi, t)$ is discretized by an upwind difference. An example of $g$ is the upwind numerical flux considered in 4, where $g(u, v, t):=q(t) v+b^{\mathrm{EO}}(u, v)$, and $b^{\mathrm{EO}}$ is the Engquist-Osher [15] numerical flux

$$
b^{\mathrm{EO}}(u, v):=b(0)+\int_{0}^{u} \max \left\{b^{\prime}(s), 0\right\} d s+\int_{0}^{v} \min \left\{b^{\prime}(s), 0\right\} d s .
$$

We assume that the parameters $\Delta t$ and $\Delta x$ satisfy the CFL condition

$$
2 \lambda \max _{t \in[0, T]}\left\|\partial_{\phi} f(., t)\right\|_{\infty} \leq 1 .
$$

2.3. Compactness criterion. We pass to the limit for $\Delta x \rightarrow 0, \Delta t \rightarrow 0$ by appealing to the embedding of $L^{\infty}\left(Q_{T}\right) \cap B V\left(Q_{T}\right)$ in $L^{1}\left(Q_{T}\right)$ (see [17]) and using the following well-known $L^{1}$ compactness criterion.

Lemma 2.1. Let $\left\{z_{h}\right\}_{h>0}$ be a sequence of functions defined on $Q_{T}$. Assume that there exist constants $C_{0}, \ldots, C_{3}$ which may depend on $T$, but not on $h$, such that

$$
\begin{gathered}
\left\|z_{h}\right\|_{L^{\infty}\left(Q_{T}\right)} \leqslant C_{0}, \quad\left\|z_{h}\right\|_{L^{1}\left(Q_{T}\right)} \leq C_{1}, \\
\left\|z_{h}(\cdot+y, \cdot)-z_{h}(\cdot, \cdot)\right\|_{L^{1}\left(Q_{T}\right)} \leq C_{2}(|y|+h) \quad \text { for all } y \in \mathbb{R} \text { as } h \downarrow 0, \\
\left\|z_{h}(\cdot, \cdot+\tau)-z_{h}(\cdot, \cdot)\right\|_{L^{1}\left(Q_{T}\right)} \leq C_{3}(\tau+h) \quad \text { for all } \tau>0 \text { as } h \downarrow 0 .
\end{gathered}
$$


Then $\left\{z_{h}\right\}_{h>0}$ is compact in the strong topology of $L_{\text {loc }}^{1}\left(Q_{T}\right)$. Moreover, any limit point of $\left\{z_{h}\right\}_{h>0}$ belongs to $L^{1}\left(Q_{T}\right) \cap L^{\infty}\left(Q_{T}\right) \cap C\left([0, T], L^{1}(I)\right)$.

2.4. Mollifiers and related functions. Let $\omega \in C_{0}^{\infty}(\mathbb{R})$ satisfy $\omega \geqslant 0, \operatorname{supp} \omega \subset$ $(-1,1)$, and $\|\omega\|_{L^{1}(\mathbb{R})}=1$. A standard mollifier with support in $(-h, h), h>0$, is then defined by $\omega_{h}(x):=\omega(x / h) / h$. For $h>0$, we define the functions

$$
\rho_{h}(x):=\int_{-\infty}^{x} \omega_{h}(\xi) d \xi, \quad \mu_{h}(x):=1-\rho_{h}(x-2 h), \quad \nu_{h}(x):=\rho_{h}(x-(1-2 h)),
$$

which satisfy $\left|\mu_{h}^{\prime}(x)\right|,\left|\nu_{h}^{\prime}(x)\right| \leqslant C_{\mu} / h$ and $\left|\mu_{h}^{\prime \prime}(x)\right|,\left|\nu_{h}^{\prime \prime}(x)\right| \leqslant C_{\mu} / h^{2}$ with a constant $C_{\mu}$ that is independent of $h$, and which have the following property (see [2]).

Lemma 2.2. Let $v \in L^{1}\left(\mathcal{T} ; L^{\infty}(I)\right)$. If the traces $\gamma_{0} v:=(\gamma v)(0, t)$ and $\gamma_{1} v:=$ $(\gamma v)(1, t)$ exist a.e. in $\mathcal{T}$, then we have for $\varphi \in C^{\infty}\left(Q_{T}\right)$,

$\lim _{h \downarrow 0} \iint_{Q_{T}} \partial_{x}\left(\varphi(x, t)\left(\mu_{h}(x)+\nu_{h}(x)\right)\right) v(x, t) d t d x=\int_{0}^{T}\left(\varphi(1, t) \gamma_{1} v-\varphi(0, t) \gamma_{0} v\right) d t$.

\section{WELL-POSEDNESS AND MONOTONICITY OF THE NUMERICAL SCHEME}

We now show that the scheme is well defined, which means that for a given vector $\phi^{n}:=\left(\phi_{0}^{n}, \ldots, \phi_{J}^{n}\right) \in\left[0, \phi_{\max }\right]^{J+1}$ at time $t^{n}$, the nonlinear equations (2.6) always have a unique solution $\phi^{n+1}$. To show this, we first assume that (2.6) has a solution, and show (in Lemma 3.1) that the scheme is monotone and (in Lemma 3.2) that it satisfies an $L^{\infty}$ stability property. These properties allow us to apply a topological degree argument to conclude that (2.6) indeed has a unique solution; see Lemma 3.3 .

3.1. Monotonicity and $L^{\infty}$ stability. First, we prove the stability and some regularity properties of the approximate solution values $\left\{\phi_{j}^{n}\right\}:=\left\{\phi_{j}^{n}: 0 \leqslant j \leqslant\right.$ $J, 0 \leqslant n \leqslant N\}$. We mainly follow the the classical work of Crandall and Majda [12] and its generalizations given by Karlsen et al. in [18, 19, 22.

Lemma 3.1. The semi-implicit scheme (2.6) is monotone under the CFL condition (2.7). This means that if we can solve (2.6) for all $n \in \mathbb{N}$, then there exist functions $\mathcal{H}_{0}, \ldots, \mathcal{H}_{J}$ such that $\phi_{j}^{n+1}=\mathcal{H}_{j}\left(\phi_{0}^{n}, \phi_{1}^{n}, \ldots, \phi_{J}^{n}, t^{n}\right)$ for $j=0, \ldots, J$, where all functions $\mathcal{H}_{0}, \ldots, \mathcal{H}_{J}$ are increasing in each $\phi$-argument.

Proof. We wish to apply the Crandall-Tartar lemma [12, 13, to conclude that the scheme is monotone. A straightforward calculation based on (2.6) yields

$$
\sum_{j=0}^{J} \phi_{j}^{n+1}=\sum_{j=0}^{J} \phi_{j}^{n}+\delta\left(t^{n}, \phi_{0}^{n}\right), \quad \delta(t, \phi):=\lambda(q(t) \phi-\Psi(t)) .
$$

Now let $\phi=\left(\phi_{0}, \ldots, \phi_{J}\right)$, and let us define the family of operators $T(t): \mathbb{R}^{J+2} \rightarrow$ $\mathbb{R}^{J+2}, t \geqslant 0$, by $(T(t))(\phi, u):=\left(\mathcal{H}_{0}(\phi, t), \ldots, \mathcal{H}_{J}(\phi, t), u-\delta\left(t, \phi_{0}\right)\right)$. We may now describe our scheme by setting $u^{0}:=0$ and defining

$$
\begin{aligned}
\left(\phi^{n+1}, u^{n+1}\right)=\left(T\left(t^{n}\right)\right)\left(\phi^{n}, u^{n}\right) & =\left(\mathcal{H}_{0}\left(\phi^{n}, t^{n}\right), \ldots, \mathcal{H}_{J}\left(\phi^{n}, t^{n}\right), u-\delta\left(t^{n}, \phi_{0}^{n}\right)\right) \\
& =\left(\phi_{0}^{n+1}, \ldots, \phi_{J}^{n+1}, u^{n}-\delta\left(t^{n}, \phi_{0}^{n}\right)\right) .
\end{aligned}
$$


We address by $T_{0}(t), \ldots, T_{J+1}(t)$ the components of $T(t)$. Then (3.1) implies

$$
\sum_{j=0}^{J+1}\left(T_{j}\left(t^{n}\right)\right)\left(\phi^{n}, u^{n}\right)=\sum_{j=0}^{J} \phi_{j}^{n}+\delta\left(t^{n}, \phi_{0}^{n}\right)+u^{n}-\delta\left(t^{n}, \phi_{0}^{n}\right)=\sum_{j=0}^{J} \phi_{j}^{n}+u^{n} .
$$

Since $t^{n}$ can be chosen arbitrarily, all operators $T(t)$ are conservative. On the other hand, let us denote by $\psi^{n}:=\left(\psi_{0}^{n}, \ldots, \psi_{j}^{n}\right)$ the second solution vector, and by $u_{\phi}^{n}$ and $u_{\psi}^{n}$ the value of $u^{n}$ associated with $\phi$ and $\psi$, respectively.

Let us define $\mathcal{D}_{j}^{n}:=\psi_{j}^{n}-\phi_{j}^{n}$ for $j=0, \ldots, J$. Then we obtain from (2.6)

$$
\begin{aligned}
\left(1+\theta_{0}\right) \mathcal{D}_{0}^{n+1}= & \left(1-\beta_{1 / 2}+\lambda q\left(t^{n}\right)\right) \mathcal{D}_{0}^{n}+\alpha_{1 / 2} \mathcal{D}_{1}^{n}+\theta_{1} \mathcal{D}_{1}^{n+1}, \\
\left(1+2 \theta_{j}\right) \mathcal{D}_{j}^{n+1}= & \left(1-\beta_{j+1 / 2}-\alpha_{j-1 / 2}\right) \mathcal{D}_{j}^{n}+\alpha_{j+1 / 2} \mathcal{D}_{j+1}^{n}+\beta_{j-1 / 2} \mathcal{D}_{j-1}^{n} \\
& +\theta_{j+1} \mathcal{D}_{j+1}^{n+1}+\theta_{j-1} \mathcal{D}_{j-1}^{n+1}, \quad j=1, \ldots, J-1, \\
\left(1+\theta_{J}\right) \mathcal{D}_{J}^{n+1}= & \left(1-\alpha_{J-1 / 2}\right) \mathcal{D}_{J}^{n}+\beta_{J-1 / 2} \mathcal{D}_{J-1}^{n}+\theta_{J-1} \mathcal{D}_{J-1}^{n+1},
\end{aligned}
$$

where, using $\left(b^{\prime}\right)^{+}(u):=\max \left\{b^{\prime}(u), 0\right\}$ and $\left(b^{\prime}\right)^{-}(u):=\min \left\{b^{\prime}(u), 0\right\}$, we define

$$
\begin{aligned}
\alpha_{j+1 / 2} & :=-\lambda\left[q\left(t^{n}\right)+\int_{0}^{1}\left(b^{\prime}\right)^{-}\left(\phi_{j+1}^{n}+\sigma\left(\psi_{j+1}^{n}-\phi_{j+1}^{n}\right)\right) d \sigma\right] \geqslant 0, j=0, \ldots, J-1, \\
\beta_{j+1 / 2} & :=\lambda \int_{0}^{1}\left(b^{\prime}\right)^{+}\left(\phi_{j+1}^{n}+\sigma\left(\psi_{j+1}^{n}-\phi_{j+1}^{n}\right)\right) d \sigma \geqslant 0, \quad j=0, \ldots, J-1, \\
\theta_{j} & := \begin{cases}\mu\left(A\left(\psi_{j}^{n+1}\right)-A\left(\phi_{j}^{n+1}\right)\right) / \mathcal{D}_{j}^{n+1} \geqslant 0 & \text { if } \mathcal{D}_{j}^{n+1} \neq 0, \quad j=0, \ldots, J . \\
0 & \text { otherwise, }\end{cases}
\end{aligned}
$$

Due to (2.7), all coefficients in (3.2) are nonnegative, which implies

$$
\begin{aligned}
\left(1+\theta_{0}\right)\left|\mathcal{D}_{0}^{n+1}\right| \leqslant & \left(1-\beta_{1 / 2}+\lambda q\left(t^{n}\right)\right)\left|\mathcal{D}_{0}^{n}\right|+\alpha_{1 / 2}\left|\mathcal{D}_{1}^{n}\right|+\theta_{1}\left|\mathcal{D}_{1}^{n+1}\right|, \\
\left(1+2 \theta_{j}\right)\left|\mathcal{D}_{j}^{n+1}\right| \leqslant & \left(1-\beta_{j+1 / 2}-\alpha_{j-1 / 2}\right)\left|\mathcal{D}_{j}^{n}\right|+\alpha_{j+1 / 2}\left|\mathcal{D}_{j+1}^{n}\right|+\beta_{j-1 / 2}\left|\mathcal{D}_{j-1}^{n}\right| \\
& +\theta_{j+1}\left|\mathcal{D}_{j+1}^{n+1}\right|+\theta_{j-1}\left|\mathcal{D}_{j-1}^{n+1}\right|, \quad j=1, \ldots, J-1, \\
\left(1+\theta_{J}\right)\left|\mathcal{D}_{J}^{n+1}\right| \leqslant & \left(1-\alpha_{J-1 / 2}\right)\left|\mathcal{D}_{J}^{n}\right|+\beta_{J-1 / 2}\left|\mathcal{D}_{J-1}^{n}\right|+\theta_{J-1}\left|\mathcal{D}_{J-1}^{n+1}\right| .
\end{aligned}
$$

Summation over these inequalities, cancelling terms wherever possible, and rewriting the result again in terms of $\left\{\phi_{j}^{n}\right\}$ and $\left\{\psi_{j}^{n}\right\}$, we obtain the inequality

$$
\sum_{j=0}^{J}\left|\psi_{j}^{n+1}-\phi_{j}^{n+1}\right| \leqslant \lambda q\left(t^{n}\right)\left|\psi_{0}^{n}-\phi_{0}^{n}\right|+\sum_{j=0}^{J}\left|\psi_{j}^{n}-\phi_{j}^{n}\right| .
$$

Then in light of (3.3), a straightforward calculation yields

$$
\sum_{j=0}^{J+1}\left|\left(T_{j}(t)\right)\left(\boldsymbol{\psi}^{n}, u_{\psi}^{n}\right)-\left(T_{j}(t)\right)\left(\phi^{n}, u_{\phi}^{n}\right)\right| \leqslant \sum_{j=0}^{J}\left|\psi_{j}^{n}-\phi_{j}^{n}\right|+\left|u_{\psi}^{n}-u_{\phi}^{n}\right|,
$$

which implies that for each $t=t^{n}$, the operator $T(t)$ is $L^{1}$ contractive. The Crandall-Tartar lemma then yields that $T(t)$ is monotone.

To conclude that the scheme (2.6) is monotone, let us choose $\phi^{0}$ and $\psi^{0}$ such that $\phi_{j}^{0} \leqslant \psi_{j}^{0}$ for $j=0, \ldots, J$ and $u_{\phi}^{0} \leqslant u_{\psi}^{0}$. Then $\phi_{j}^{1} \leqslant \psi_{j}^{1}$ for $j=0, \ldots, J$ and $u_{\psi}^{1} \geqslant u_{\phi}^{1}$ due to the monotonicity, i.e., the scheme (2.6) is monotone for the first time step. Repeating this argument, we conclude that the scheme is monotone for each following time step, and therefore monotone. 
Lemma 3.2. Assume that for any given vector $\phi^{n} \in\left[0, \phi_{\max }\right]^{J+1}$, there exists a solution $\phi^{n+1}$ of the nonlinear equations (2.6). Then $\phi^{n+1} \in\left[0, \phi_{\max }\right]^{J+1}$. In other words, the scheme (2.6) is $L^{\infty}$ stable.

Proof. We rewrite the semi-implicit scheme (2.6) as a nonlinear system

$$
\mathbf{s}\left(\phi^{n+1}\right)=\mathbf{w}^{n}\left(\phi^{n}\right)
$$

where the vectors $\mathbf{s}$ and $\mathbf{w}$ are given by

$$
\begin{aligned}
w_{0}^{n} & =\phi_{0}^{n}-\lambda g_{1 / 2}^{n}+\lambda q\left(t^{n}\right) \phi_{0}^{n}, \quad w_{J}^{n}=\phi_{J}^{n}+\lambda g_{J-1 / 2}^{n}-\lambda \Psi\left(t^{n}\right), \\
w_{j}^{n} & =\phi_{j}^{n}-\lambda\left(g_{j+1 / 2}^{n}-g_{j-1 / 2}^{n}\right), \quad j=1, \ldots, J-1, \\
s_{0} & =\phi_{0}^{n+1}-\mu d_{1 / 2}^{n+1}, \quad s_{J}=\phi_{j}^{n+1}+\mu d_{J-1 / 2}^{n+1}, \\
s_{j} & =\phi_{J}^{n+1}-\mu\left(d_{j+1 / 2}^{n+1}-d_{j-1 / 2}^{n+1}\right), \quad j=1, \ldots, J-1 .
\end{aligned}
$$

To establish the desired maximum principle, we rewrite (3.5) in the form

$$
\mathbf{M}\left(\phi^{n+1}\right) \phi^{n+1}=\mathbf{w}^{n}\left(\phi^{n}\right)
$$

where, in terms of

$$
D_{j+1 / 2}^{n+1}:= \begin{cases}\frac{A\left(\phi_{j+1}^{n+1}\right)-A\left(\phi_{j}^{n+1}\right)}{\phi_{j+1}^{n+1}-\phi_{j}^{n+1}} & \text { if } \phi_{j+1}^{n+1} \neq \phi_{j}^{n+1}, \quad j=0, \ldots, J-1, \\ 0 & \text { otherwise, }\end{cases}
$$

the entries $\left(m_{i, j}\right)_{0 \leqslant i, j \leqslant J}$ of the tridiagonal matrix $\mathbf{M}=\mathbf{M}(\phi)$ are given by

$$
\begin{aligned}
m_{j, j-1} & =-\mu D_{j-1 / 2}^{n+1}, \quad j=1, \ldots, J, \\
m_{j j} & =1+\mu D_{j+1 / 2}^{n+1}+\mu D_{j-1 / 2}^{n+1}, \quad j=1, \ldots, J-1, \\
m_{j, j+1} & =-\mu D_{j+1 / 2}^{n+1}, \quad j=0, \ldots, J-1, \\
m_{00} & =1+\mu D_{1 / 2}, \quad m_{J J}=1+\mu D_{J-1 / 2} .
\end{aligned}
$$

Since the matrix $\mathbf{M}(\phi)$ is monotone for all $\phi \in \mathbb{R}^{J+1}$, the elements of its inverse $\mathbf{M}^{-1}$ are nonnegative. Furthermore, we have $\left\|\mathbf{M}^{-1}\right\|_{\infty} \leq 1$ since

$$
m_{i i}-\sum_{\substack{i, j=0 \\ i \neq j}}^{J}\left|m_{i j}\right| \geq 1, \quad i=0, \ldots, J
$$

Now consider the initial data $\tilde{\varphi}_{j}^{0}:=0$ for $i=0, \ldots, J$. Noting that $\mathbf{w}^{0}(0, \ldots, 0)=$ $\left(0, \ldots, 0,-\lambda \Psi\left(t^{0}\right)\right)^{\mathrm{T}}$, we conclude by the nonnegativity of $\mathbf{M}^{-1}$ that $\phi_{j}^{1} \geqslant 0$ for $j=0, \ldots, J$. On the other hand, if we start from $\bar{\varphi}_{j}^{0}:=\phi_{\max }$ for $i=0, \ldots, J$, then due to (H4), the components of

$$
\mathbf{w}^{0}\left(\phi_{\max }, \ldots, \phi_{\max }\right)=\left(\phi_{\max }, \ldots, \phi_{\max }, \lambda\left(q(t) \phi_{\max }-\Psi\left(t^{0}\right)\right)\right)^{\mathrm{T}}
$$

are all smaller than or equal to $\phi_{\max }$, so that $\left\|\mathbf{M}^{-1}\right\|_{\infty} \leq 1$ implies that $\phi_{j}^{1} \leqslant \phi_{\max }$ for $j=0, \ldots, J$. Since the scheme is monotone, an arbitrary initial datum with $\tilde{\varphi}_{j}^{0} \leqslant \phi_{j}^{0} \leqslant \bar{\varphi}_{j}^{0}$ for $j=0, \ldots, J$ will lead to solution values $\left\{\phi_{j}^{1}\right\}$ after the first time step with $\tilde{\varphi}_{j}^{1} \leqslant \phi_{j}^{1} \leqslant \bar{\varphi}_{j}^{1}$ for $j=0, \ldots, J$, and therefore $0 \leqslant \phi_{j}^{1} \leqslant \phi_{\max }$ for $j=0, \ldots, J$. Repeating the same argument for each successive time step shows that $0 \leqslant \tilde{\varphi}_{j}^{n} \leqslant \phi_{j}^{n} \leqslant \bar{\varphi}_{j}^{n} \leqslant \phi_{\max }$ for $j=0, \ldots, J, n=1,2,3, \ldots$. 


\subsection{Existence and uniqueness.}

Lemma 3.3. Let $\phi^{0} \in\left[0, \phi_{\max }\right]^{J+1}$, and assume that the CFL condition (2.7) is satisfied. Then the scheme (2.6) is well defined:

a) There exists a unique solution $\left\{\phi_{j}^{n}\right\} \subset\left[0, \phi_{\max }\right]$ to the nonlinear equations defined by the numerical scheme (2.6).

b) The scheme (2.6) defines an $L^{1}$-contractive solution operator. This means that if $\left\{\phi_{j}^{n}\right\}$ and $\left\{\psi_{j}^{n}\right\}$ are numerical solutions associated with the initial values $\phi_{0}^{0}, \ldots, \phi_{J}^{0}$ and $\psi_{0}^{0}, \ldots, \psi_{j}^{0}$, respectively, then

$$
\sum_{j=0}^{J}\left|\psi_{j}^{n+1}-\phi_{j}^{n+1}\right| \leqslant \sum_{j=0}^{J}\left|\psi_{j}^{n}-\phi_{j}^{n}\right|, \quad n=0,1,2, \ldots
$$

In particular, choosing $\phi_{j}^{n}=\psi_{j}^{n}$ for $j=0, \ldots, J$, we see that $\phi^{n+1}$ is uniquenely defined by (2.6).

Proof. Let $\phi^{n} \in\left[0, \phi_{\max }\right]^{J+1}$. In order to prove the existence of $\phi^{n+1}$, we adopt an argument used in 20 to prove the existence of the solution of implicit schemes for hyperbolic equations, based on topological degree theory (see [14 for more details). To this end, the numerical scheme (2.6) can be written in the form

$$
\phi^{n+1}-\mathcal{G}\left(\phi^{n+1}\right)=\tilde{\mathcal{H}}\left(\phi^{n}, t^{n}\right)
$$

where $\tilde{\mathcal{H}}\left(\cdot, t^{n}\right):=\left(\tilde{\mathcal{H}}_{0}\left(\cdot, t^{n}\right), \ldots, \tilde{\mathcal{H}}_{J}\left(\cdot, t^{n}\right)\right)^{\mathrm{T}}$ and $\mathcal{G}=\left(\mathcal{G}_{0}, \ldots, \mathcal{G}_{J}\right)^{\mathrm{T}}$ are continuous maps from $\mathbb{R}^{J+1}$ into $\mathbb{R}^{J+1}$ given by

$$
\begin{aligned}
\tilde{\mathcal{H}}_{0}\left(\phi, t^{n}\right) & :=\phi_{0}-\lambda\left(g\left(\phi_{0}, \phi_{1}, t^{n}\right)-q\left(t^{n}\right) \phi_{0}\right), \\
\tilde{\mathcal{H}}_{j}\left(\phi, t^{n}\right) & :=\phi_{j}-\lambda\left(g\left(\phi_{j}, \phi_{j+1}, t^{n}\right)-g\left(\phi_{j-1}, \phi_{j}, t^{n}\right)\right), \quad j=1, \ldots, J-1, \\
\tilde{\mathcal{H}}_{J}\left(\phi, t^{n}\right) & :=\phi_{J}-\lambda\left(\Psi\left(t^{n}\right)-g\left(\phi_{J-1}, \phi_{J}, t^{n}\right)\right), \\
\mathcal{G}_{0}(\phi) & :=\mu\left(A\left(\phi_{1}\right)-A\left(\phi_{0}\right)\right), \\
\mathcal{G}_{j}(\phi) & :=\mu\left(A\left(\phi_{j+1}\right)-2 A\left(\phi_{j}\right)+A\left(\phi_{j-1}\right)\right), \quad j=1, \ldots, J-1, \\
\mathcal{G}_{J}(\phi) & :=-\mu\left(A\left(\phi_{J}\right)-A\left(\phi_{J-1}\right)\right) .
\end{aligned}
$$

We recall that due to Lemma 3.2, if $\phi^{n+1}$ satisfies (3.10), then $\phi^{n+1} \in\left[0, \phi_{\max }\right]^{J+1}$. On the other hand, by applying Lemma 3.2 to the special case $A \equiv 0$, it is clear that under the CFL condition (2.7), the explicit scheme for the hyperbolic case

$$
\phi^{n+1}=\tilde{\mathcal{H}}\left(\phi^{n}, t^{n}\right)
$$

satisfies the same $L^{\infty}$ bound, i.e., we also have $\mathcal{H}_{j}\left(\phi^{n}, t^{n}\right) \in\left[0, \phi_{\max }\right]$. Therefore, if $B_{R} \subset \mathbb{R}^{J+1}$ is a ball with center 0 and a suffiently large radius $R$, then (3.10) has no solution on the boundary of $B_{R}$, and one can define the topological degree of the mapping Id- $\mathcal{G}$ associated with the set $B_{R}$ and the point $\tilde{\mathcal{H}}\left(\phi^{n}, t^{n}\right)$, that is, $\operatorname{deg}(\operatorname{Id}-$ $\left.\mathcal{G}, B_{R}, \tilde{\mathcal{H}}\left(\phi^{n}, t^{n}\right)\right)$. Furthermore, if $\alpha \in[0,1]$, then the same argument allows us to define $\operatorname{deg}\left(\operatorname{Id}-\alpha \mathcal{G}, B_{R}, \tilde{\mathcal{H}}\left(\phi^{n}, t^{n}\right)\right)$. Then the property of invariance of the degree under continuous transformations asserts that $\operatorname{deg}\left(\operatorname{Id}-\alpha \mathcal{G}, B_{R}, \tilde{\mathcal{H}}\left(\phi^{n}, t^{n}\right)\right)$ does not depend on $\alpha$. This gives

$$
\operatorname{deg}\left(\operatorname{Id}-\mathcal{G}, B_{R}, \tilde{\mathcal{H}}\left(\phi^{n}, t^{n}\right)\right)=\operatorname{deg}\left(\operatorname{Id}, B_{R}, \tilde{\mathcal{H}}\left(\phi^{n}, t^{n}\right)\right)=1 .
$$

The equality for $\alpha=0$ holds since we can solve the scheme (3.11) a unique way.

Since $q\left(t^{n}\right) \leqslant 0$, inequality (3.3) implies (3.9). This concludes the proof. 


\section{Estimates on the APPROXimate SOLUtions}

In the remainder of the paper, it is always understood that the numbered constants $C_{4}, C_{5}, \ldots$ are independent of $\Delta:=(\Delta x, \Delta t)$.

\section{1. $B V$ estimates.}

Lemma 4.1. Under the assumptions of Lemma 3.2, there exists a constant $C_{4}$ such that

$$
\sum_{j=0}^{J-1}\left|\phi_{j+1}^{n}-\phi_{j}^{n}\right| \leq \sum_{j=0}^{J-1}\left|\phi_{j+1}^{0}-\phi_{j}^{0}\right|+C_{4} .
$$

Proof. We define $w_{j+1 / 2}^{n}:=\phi_{j+1}^{n}-\phi_{j}^{n}$ for $j=0, \ldots, J-1$ and introduce the following notation. For $j=0, \ldots, J-1$, we set

$$
\begin{gathered}
B_{j+1 / 2}^{n}:=\frac{g\left(\phi_{j}^{n}, \phi_{j+1}^{n}, t^{n}\right)-g\left(\phi_{j}^{n}, \phi_{j}^{n}, t^{n}\right)}{\phi_{j+1}^{n}-\phi_{j}^{n}}, \\
C_{j+1 / 2}^{n}:=\frac{g\left(\phi_{j}^{n}, \phi_{j+1}^{n}, t^{n}\right)-g\left(\phi_{j+1}^{n}, \phi_{j+1}^{n}, t^{n}\right)}{\phi_{j}^{n}-\phi_{j+1}^{n}}, \quad D_{j+1 / 2}^{n}:=\frac{d_{j+1 / 2}^{n}}{\phi_{j+1}^{n}-\phi_{j}^{n}}
\end{gathered}
$$

if $\phi_{j+1}^{n} \neq \phi_{j}^{n}$, and $B_{j+1 / 2}^{n}=C_{j+1 / 2}^{n}=D_{j+1 / 2}^{n}=0$ otherwise. Then we define

$$
\begin{aligned}
\bar{A}_{j+1 / 2} & :=\lambda C_{j-1 / 2}^{n}, \quad j=1, \ldots, J, \\
\bar{B}_{j+1 / 2} & :=1-\lambda\left(C_{j+1 / 2}^{n}-B_{j+1 / 2}^{n}\right), \quad j=0, \ldots, J-1, \\
\bar{C}_{j+1 / 2}^{n} & :=-\lambda B_{j+3 / 2}^{n}, \quad j=-1, \ldots, J-2 .
\end{aligned}
$$

With these definitions, we can write the interior scheme (2.6b) as

$$
\begin{aligned}
w_{j+1 / 2}^{n+1}\left(1+2 \mu D_{j+1 / 2}^{n+1}\right)= & \bar{A}_{j+1}^{n} w_{j-1 / 2}^{n}+\bar{B}_{j+1 / 2}^{n} w_{j+1 / 2}^{n} \\
& +\bar{C}_{j+1 / 2}^{n} w_{j+3 / 2}^{n}+\mu D_{j-1 / 2}^{n+1} w_{j-1 / 2}^{n+1} \\
& +\mu D_{j+3 / 2}^{n+1} w_{j+3 / 2}^{n+1}, \quad j=1, \ldots, J-2,
\end{aligned}
$$

while the boundary schemes (2.6a) and (2.6c) take the respective forms

$$
\begin{aligned}
w_{1 / 2}^{n+1}= & \lambda b\left(\phi_{0}^{n}\right)+\bar{B}_{1 / 2}^{n} w_{1 / 2}^{n}+\bar{C}_{1 / 2}^{n} w_{3 / 2}^{n}-2 \mu D_{1 / 2}^{n+1} w_{1 / 2}^{n+1}+\mu D_{3 / 2}^{n+1} w_{3 / 2}^{n+1}, \\
w_{J-1 / 2}^{n+1}= & \bar{A}_{J-1 / 2}^{n} w_{J-3 / 2}^{n}+\bar{B}_{J-1 / 2}^{n} w_{J-1 / 2}^{n}+\lambda\left(f\left(\phi_{J}^{n}, t\right)-\Psi\left(t^{n}\right)\right) \\
& +\mu D_{J-3 / 2}^{n+1} w_{J-3 / 2}^{n+1}-2 \mu D_{J-1 / 2}^{n+1} w_{J-1 / 2}^{n+1} .
\end{aligned}
$$

Noting that

$\bar{A}_{j+1 / 2}^{n}, \bar{B}_{j+1 / 2}^{n}, \bar{C}_{j+1 / 2}^{n} \geqslant 0, \quad \bar{A}_{j+3 / 2}^{n}+\bar{B}_{j+1 / 2}^{n}+\bar{C}_{j-1 / 2}^{n}=1, \quad j=0, \ldots, J-1$, we get from (4.4)-4.6

$$
\begin{aligned}
& \sum_{j=0}^{J-1}\left|w_{j+1 / 2}^{n+1}\right|\left(1+2 \mu D_{j+1 / 2}^{n+1}\right) \\
& \leqslant\left|\lambda b\left(\phi_{0}^{n}\right)\right|+\left(\bar{B}_{1 / 2}^{n}+\bar{A}_{3 / 2}^{n}\right)\left|w_{1 / 2}^{n}\right|+\left(\bar{B}_{J-1 / 2}^{n}+\bar{C}_{J-3 / 2}^{n}\right)\left|w_{J-1 / 2}^{n}\right| \\
& \quad+\lambda\left|f\left(\phi_{J}^{n}, t^{n}\right)-\Psi\left(t^{n}\right)\right|+\sum_{j=1}^{J-2}\left|w_{j+1 / 2}^{n}\right|+2 \mu \sum_{j=0}^{J-1} D_{j+1 / 2}^{n+1}\left|w_{j+1 / 2}^{n+1}\right| \\
& \quad-\mu D_{1 / 2}^{n+1}\left|w_{1 / 2}^{n+1}\right|-\mu D_{J-1 / 2}^{n+1}\left|w_{J-1 / 2}^{n+1}\right| .
\end{aligned}
$$


Next, we rewrite the boundary condition (2.6a) as

$$
\phi_{0}^{n+1}=\phi_{0}^{n}+\bar{C}_{-1 / 2}^{n} w_{1 / 2}^{n}+\mu D_{1 / 2}^{n+1} w_{1 / 2}^{n+1}-\lambda b\left(\phi_{0}^{n}\right) .
$$

Since $b\left(\phi_{0}^{n}\right) \leqslant 0$, we get

$$
\begin{aligned}
\left|\lambda b\left(\phi_{0}^{n}\right)\right|-\mu D_{1 / 2}^{n+1}\left|w_{1 / 2}^{n+1}\right| & =\phi_{0}^{n+1}-\phi_{0}^{n}-\bar{C}_{-1 / 2}^{n} w_{1 / 2}^{n}-\mu D_{1 / 2}^{n+1}\left(w_{1 / 2}^{n+1}+\left|w_{1 / 2}^{n+1}\right|\right) \\
& \leqslant \phi_{0}^{n+1}-\phi_{0}^{n}+\bar{C}_{-1 / 2}^{n}\left|w_{1 / 2}^{n}\right| .
\end{aligned}
$$

Inserting this into (4.7), we obtain

$$
\begin{aligned}
\sum_{j=0}^{J-1}\left|w_{j+1 / 2}^{n+1}\right| \leqslant & \phi_{0}^{n+1}-\phi_{0}^{n}+\sum_{j=0}^{J-1}\left|w_{j+1 / 2}^{n}\right|+\lambda\left|f\left(\phi_{J}^{n}, t^{n}\right)-\Psi\left(t^{n}\right)\right| \\
& -\bar{A}_{J+1 / 2}^{n}\left|w_{J-1 / 2}^{n}\right|-\mu D_{J-1 / 2}^{n+1}\left|w_{J-1 / 2}^{n+1}\right| .
\end{aligned}
$$

From boundary condition (2.6c) we obtain

$$
\phi_{J}^{n+1}=\phi_{J}^{n}-\bar{A}_{J+1 / 2}^{n} w_{J-1 / 2}^{n}-\mu D_{J-1 / 2}^{n+1} w_{J-1 / 2}^{n+1}+\lambda\left(f\left(\phi_{J}^{n}, t^{n}\right)-\Psi\left(t^{n}\right)\right) .
$$

We will now finish the proof for case (b) of (H6), and then deal with the simpler case (a). For case (b), (4.10) implies

$$
\begin{aligned}
\lambda\left(f\left(\phi_{J}^{n}, t^{n}\right)-\Psi\left(t^{n}\right)\right)= & \phi_{J}^{n}-\phi_{J}^{n-1}+\bar{A}_{J+1 / 2}^{n-1} w_{J-1 / 2}^{n-1}+\mu D_{J-1 / 2}^{n} w_{J-1 / 2}^{n} \\
& +\lambda\left(f\left(\phi_{J}^{n}, t^{n}\right)-f\left(\phi_{J}^{n-1}, t^{n-1}\right)\right)-\lambda\left(\Psi\left(t^{n}\right)-\Psi\left(t^{n-1}\right)\right)
\end{aligned}
$$

for $n=1, \ldots, N$. We now define $\Theta(\phi, t):=\phi+\lambda f(\phi, t)$; note that due to (2.7), $\Theta(\cdot, t)$ is a strictly increasing function for all $t \in \mathcal{T}$. Then the last equation implies

$$
\begin{aligned}
\lambda\left|f\left(\phi_{J}^{n}, t^{n}\right)-\Psi\left(t^{n}\right)\right| \leqslant & \operatorname{sgn}\left(f\left(\phi_{J}^{n}, t^{n}\right)-\Psi\left(t^{n}\right)\right)\left(\Theta\left(\phi_{J}^{n}, t^{n}\right)-\Theta\left(\phi_{J}^{n-1}, t^{n-1}\right)\right) \\
& +\bar{A}_{J+1 / 2}^{n-1}\left|w_{J-1 / 2}^{n-1}\right|+\mu D_{J-1 / 2}^{n}\left|w_{J-1 / 2}^{n}\right| \\
& +\lambda\left|\Psi\left(t^{n}\right)-\Psi\left(t^{n-1}\right)\right|, \quad i=1, \ldots, N .
\end{aligned}
$$

Inserting this into (4.9), we get for $M=2, \ldots, N$

$$
\begin{aligned}
\sum_{j=0}^{J-1}\left|w_{j+1 / 2}^{M}\right| \leqslant & \phi_{0}^{M}-\phi_{0}^{1}+\sum_{j=0}^{J-1}\left|w_{j+1 / 2}^{1}\right|+\bar{A}_{J+1 / 2}^{0}\left|w_{J-1 / 2}^{0}\right| \\
& +\mu D_{J-1 / 2}^{1}\left|w_{J-1 / 2}^{0}\right|+\lambda \sum_{n=1}^{M}\left|\Psi\left(t^{n}\right)-\Psi\left(t^{n-1}\right)\right|+S \\
S:= & \sum_{n=1}^{M} \sigma_{n}\left(\Theta\left(\phi_{J}^{n}, t^{n}\right)-\Theta\left(\phi_{J}^{n-1}, t^{n-1}\right)\right),
\end{aligned}
$$

where $\sigma_{n}:=\operatorname{sgn}\left(f\left(\phi_{J}^{n}, t^{n}\right)-\Psi\left(t^{n}\right)\right)$ for $n=1, \ldots, N$. To estimate $S$, we define $m$ to be the smallest integer such there are numbers $n_{0}:=0, n_{1}, n_{2}, \ldots, n_{m}:=M$ with $n_{0}+1=1 \leqslant n_{1}, n_{1}+1 \leqslant n_{2}$, and so on until $n_{m-1}+1 \leqslant n_{m}$, such that $\sigma_{n} \in\{-1,0,1\}$ is constant for $n=n_{l}+1, \ldots, n_{l+1}, l=0, \ldots, m-1$.

Defining $\tau_{l}:=t^{n_{l+1}}$ for $l=0, \ldots, m-1$ and $\tilde{\sigma}_{l}:=\operatorname{sgn}\left(f\left(\phi_{J}^{n_{l+1}}, \tau_{l}\right)-\Psi\left(\tau_{l}\right)\right)$ for $l=0, \ldots, m-1$, we can rewrite $S$ as $S=S_{0}+\cdots+S_{m-1}$, where

$$
S_{l}:=\tilde{\sigma}_{l} \sum_{k=n_{l}+1}^{n_{l+1}}\left(\Theta\left(\phi_{J}^{k}, t^{k}\right)-\Theta\left(\phi_{J}^{k-1}, t^{k-1}\right)\right)=\tilde{\sigma}_{l}\left(\Theta\left(\phi_{J}^{n_{l+1}}, t^{n_{l+1}}\right)-\Theta\left(\phi_{J}^{n_{l}}, t^{n_{l}}\right)\right)
$$


for $l=0, \ldots, m-1$. Note that $\tilde{\sigma}_{l} \neq \tilde{\sigma}_{l+1}$ for $l=0, \ldots, m-2$, since otherwise we could merge two neighbouring index segments where $\sigma_{n}$ is constant to one, in contradiction to the assumption that $m$ is minimal. Clearly, $\tilde{\sigma}_{l}=0$ implies $S_{l}=0$. If $\tilde{\sigma}_{l}=1$, then $S_{l}=\Theta\left(\phi_{J}^{n_{l+1}}, t^{n_{l+1}}\right)-\Theta\left(\phi_{J}^{n_{l}}, t^{n_{l}}\right)$. However, $\tilde{\sigma}_{l}=1$ implies that $\phi_{J}^{n_{l+1}}<\varphi\left(t^{n_{l+1}}\right)$. On the other hand, if $l \geqslant 1$, then due to the minimality of $m$ we know that $\sigma_{n_{l}} \in\{0,-1\}$, and therefore $\phi_{J}^{n_{l}} \geqslant \varphi\left(t^{n_{l}}\right)$. Consequently, the monotonicity of $\Theta(\cdot, t)$ yields that $S_{l} \leqslant \Theta\left(\varphi\left(t^{n_{l+1}}\right), t^{n_{l+1}}\right)-\Theta\left(\varphi\left(t^{n_{l}}\right), t^{n_{l}}\right)$ for $l \geqslant 1$ and, if $\tilde{\sigma}_{0}=1, S_{0} \leqslant \Theta\left(\varphi\left(t^{n_{1}}\right), t^{n_{1}}\right)-\Theta\left(\phi_{J}^{0}, 0\right)$. Similarly, if $\tilde{\sigma}_{l}=-1$, we conclude that $S_{l} \leqslant-\left[\Theta\left(\varphi\left(t^{n_{l+1}}\right), t^{n_{l+1}}\right)-\Theta\left(\varphi\left(t^{n_{l}}\right), t^{n_{l}}\right)\right]$ if $l \geqslant 1$ and $S_{l} \leqslant$ $-\left[\Theta\left(\varphi\left(t^{n_{l+1}}\right), t^{n_{l+1}}\right)-\Theta\left(\phi_{J}^{0}, 0\right)\right]$ if $\tilde{\sigma}_{0}=-1$. Combining the three cases of $\tilde{\sigma}_{l}$, we get that $S_{l} \leqslant\left|\Theta\left(\varphi\left(t^{n_{l+1}}\right), t^{n_{l+1}}\right)-\Theta\left(\varphi\left(t^{n_{l}}\right), t^{n_{l}}\right)\right|$ for $l=1, \ldots, m-1$ and $S_{0} \leqslant$ $\left|\Theta\left(\varphi\left(t^{n_{1}}\right), t^{n_{1}}\right)-\Theta\left(\phi_{J}^{0}, 0\right)\right|$. This implies

$$
\begin{aligned}
S & \leqslant\left|\Theta\left(\varphi\left(t^{n_{1}}\right), t^{n_{1}}\right)-\Theta\left(\phi_{J}^{0}, 0\right)\right|+\sum_{l=0}^{m-1}\left|\Theta\left(\varphi\left(t^{n_{l+1}}\right), t^{n_{l+1}}\right)-\Theta\left(\varphi\left(t^{n_{l}}\right), t^{n_{l}}\right)\right| \\
& \leqslant \phi_{\max }+\left[1+\lambda\left(\left\|b^{\prime}\right\|_{\infty}+\|q\|_{\infty}\right)\right] \operatorname{TV}_{\left[0, t_{M}\right]}(\varphi)+\phi_{\max } \operatorname{TV}_{\left[0, t_{M}\right]}(q) .
\end{aligned}
$$

Inserting this into (4.12) and using (4.9) for $n=0$, we obtain

$$
\begin{aligned}
\sum_{j=0}^{J-1}\left|w_{j+1 / 2}^{M}\right| \leqslant & \phi_{0}^{M}-\phi_{0}^{0}+\phi_{\max }+\operatorname{TV}\left(\phi_{\Delta}^{0}\right)+\operatorname{TV}_{\mathcal{T}}(\Psi) \\
& +\left[1+\lambda\left(\left\|b^{\prime}\right\|_{\infty}+\|q\|_{\infty}\right)\right] \operatorname{TV}_{\left[0, t_{M}\right]}(\varphi)+\phi_{\max } \operatorname{TV}_{\left[0, t_{M}\right]}(q),
\end{aligned}
$$

which concludes the proof for case (b) of (H6).

In case (a) of (H6), the expression $\left|f\left(\phi_{J}^{n}, t\right)-\Psi\left(t^{n}\right)\right|$ in (4.9) is replaced by $-b\left(\phi_{J}^{n}\right)$ (since the function $b(\phi)$ is nonpositive). From (4.10) we then obtain

$$
\sum_{j=0}^{J-1}\left|w_{j+1 / 2}^{n+1}\right| \leqslant \phi_{0}^{n+1}-\phi_{0}^{n}+\phi_{J}^{n}-\phi_{J}^{n+1}+\sum_{j=0}^{J-1}\left|w_{j+1 / 2}^{n}\right|,
$$

which yields the following inequality (instead of (4.13)) for case (a) of (H6):

$$
\sum_{j=0}^{J-1}\left|w_{j+1 / 2}^{M}\right| \leqslant \phi_{0}^{M}-\phi_{0}^{0}+\phi_{J}^{0}-\phi_{J}^{M}+\operatorname{TV}\left(\phi_{\Delta}^{0}\right) .
$$

This concludes the proof of Lemma 4.1.

Lemma 4.2. There exists a constant $C_{5}$ such that

$$
\Delta x \sum_{j=0}^{J}\left|\phi_{j}^{n+1}-\phi_{j}^{n}\right| \leq C_{5} \Delta t \quad \text { for } n=0, \ldots, N-1 .
$$

Proof. We first define

$$
\hat{B}_{j}^{n+1 / 2}:=\frac{g\left(\phi_{j-1}^{n}, \phi_{j}^{n+1}, t^{n+1}\right)-g\left(\phi_{j-1}^{n}, \phi_{j}^{n}, t^{n+1}\right)}{\phi_{j}^{n+1}-\phi_{j}^{n}}
$$

if $\phi_{j}^{n+1} \neq \phi_{j}^{n}$ and $\hat{B}_{j}^{n+1 / 2}:=0$, otherwise, for $j=1, \ldots, J$,

$$
\hat{C}_{j}^{n+1 / 2}:=\frac{g\left(\phi_{j}^{n+1}, \phi_{j+1}^{n+1}, t^{n+1}\right)-g\left(\phi_{j}^{n}, \phi_{j+1}^{n+1}, t^{n+1}\right)}{\phi_{j}^{n+1}-\phi_{j}^{n}}
$$


if $\phi_{j}^{n+1} \neq \phi_{j}^{n}$ and $\hat{C}_{j}^{n+1 / 2}:=0$, otherwise, for $j=0, \ldots, J-1$, and

$$
\hat{D}_{j}^{n+1 / 2}:=\frac{A\left(\phi_{j}^{n+1}\right)-A\left(\phi_{j}^{n}\right)}{\phi_{j}^{n+1}-\phi_{j}^{n}}
$$

if $\phi_{j}^{n+1} \neq \phi_{j}^{n}$ and $\hat{D}_{j}^{n+1 / 2}:=0$, otherwise, for $j=0, \ldots, J$. From (2.6) we get

$$
\begin{aligned}
(1+ & \left.\mu \hat{D}_{0}^{n+3 / 2}\right) u_{0}^{n+3 / 2}-\mu \hat{D}_{1}^{n+3 / 2} u_{1}^{n+3 / 2} \\
= & \left(1-\lambda \hat{C}_{0}^{n+1 / 2}+\lambda q\left(t^{n+1}\right)\right) u_{0}^{n+1 / 2}-\lambda \hat{B}_{1}^{n+1 / 2} u_{1}^{n+1 / 2} \\
& +\lambda\left(q\left(t^{n+1}\right)-q\left(t^{n}\right)\right)\left(\phi_{0}^{n}-\phi_{1}^{n}\right), \\
-\mu & \hat{D}_{j-1}^{n+3 / 2} u_{j-1}^{n+3 / 2}+\left(1+2 \mu \hat{D}_{j}^{n+1 / 2}\right) u_{j}^{n+3 / 2}-\mu \hat{D}_{j+1}^{n+3 / 2} u_{j+1}^{n+3 / 2} \\
= & \lambda \hat{C}_{j-1}^{n+1 / 2} u_{j-1}^{n+1 / 2}+\left(1-\lambda \hat{C}_{j}^{n+1 / 2}+\lambda \hat{B}_{j}^{n+1 / 2}\right) u_{j}^{n+1 / 2} \\
& \quad-\lambda \hat{B}_{j+1}^{n+1 / 2} u_{j+1}^{n+1 / 2}, \quad j=1, \ldots, J-1, \\
-\mu & \hat{D}_{J-1}^{n+3 / 2} u_{J-1}^{n+3 / 2}+\left(1+\mu \hat{D}_{j}^{n+3 / 2}\right) u_{J}^{n+3 / 2} \\
= & \lambda \hat{C}_{J-1}^{n+1 / 2} u_{J-1}^{n+1 / 2}+\left(1+\lambda \hat{B}_{J}^{n+1 / 2}\right) u_{J}^{n+1 / 2}+\lambda\left(q\left(t^{n+1}\right)-q\left(t^{n}\right)\right) \phi_{J}^{n} \\
& -\lambda\left(\Psi\left(t^{n+1}\right)-\Psi\left(t^{n}\right)\right) .
\end{aligned}
$$

These equations form a linear system of equations for $u_{0}^{n+3 / 2}, \ldots, u_{J}^{n+3 / 2}$ with a monotone coefficient matrix $\hat{\mathbf{D}}$ having a nonnegative inverse $\hat{\mathbf{D}}^{-1}$. Since the entries of $\hat{D}_{i j}$ of $\hat{\mathbf{D}}$ satisfy a property analogous to (3.8), we have that $\left\|\hat{\mathbf{D}}^{-1}\right\|_{\infty} \leqslant 1$. Using the CFL condition (2.7) and a summation by parts, we deduce the inequality

$$
\begin{aligned}
\sum_{j=0}^{J}\left|u_{j}^{n+3 / 2}\right| \leqslant & \sum_{j=0}^{J}\left|u_{j}^{n+1 / 2}\right|+\lambda\left|q\left(t^{n+1}\right)-q\left(t^{n}\right)\right|\left|\phi_{0}^{n}-\phi_{1}^{n}\right| \\
& +\lambda\left|q\left(t^{n+1}\right)-q\left(t^{n}\right)\right| \phi_{J}^{n}+\lambda\left|\Psi\left(t^{n+1}\right)-\Psi\left(t^{n}\right)\right| .
\end{aligned}
$$

We can now proceed inductively to obtain

$$
\sum_{j=0}^{J}\left|u_{j}^{n+3 / 2}\right| \leqslant \sum_{j=0}^{J}\left|u_{j}^{1 / 2}\right|+2 \lambda \phi_{\max } \operatorname{TV}_{\left[0, t^{n}\right]}(q)+\lambda \operatorname{TV}_{\left[0, t^{n}\right]}(\Psi) .
$$

Finally, we show that the sum in the right-hand side is bounded. From (2.6) we get

$$
\begin{aligned}
& \left(1+\kappa_{0}\right) u_{0}^{1 / 2}-\kappa_{1} u_{1}^{1 / 2}=-\lambda g_{1 / 2}^{n}+\lambda q\left(t^{n}\right) \phi_{0}+\mu d_{1 / 2}^{0}, \\
& -\kappa_{j} u_{j-1 / 2}^{1 / 2}+\left(1+2 \kappa_{j}\right) u_{j}^{1 / 2}-\kappa_{j+1} u_{j+1}^{1 / 2} \\
& \quad=-\lambda\left(g_{j+1 / 2}^{0}-g_{j-1 / 2}^{0}\right)+\mu\left(d_{j+1 / 2}^{0}-d_{j-1 / 2}^{0}\right), \quad j=1, \ldots, J-1, \\
& -\kappa_{J-1} u_{J-1}^{1 / 2}+\left(1+\kappa_{J}\right) u_{J}^{1 / 2}=\lambda g_{J-1 / 2}^{0}-\lambda \Psi\left(t^{n}\right)-\mu d_{J-1 / 2}^{0},
\end{aligned}
$$

where we define the nonnegative numbers

$$
\kappa_{j}:= \begin{cases}\mu\left(A\left(\phi_{j}^{1}\right)-A\left(\phi_{j}^{0}\right)\right) / u_{j}^{1 / 2} & \text { if } u_{j}^{1 / 2} \neq 0, \\ 0 & \text { otherwise }\end{cases}
$$


Clearly, (4.16) is again a linear system for $u_{0}^{1 / 2}, \ldots, u_{J}^{1 / 2}$ with a monotone coefficient matrix $\mathbf{K}$ whose inverse is nonnegative with $\left\|\mathbf{K}^{-1}\right\|_{\infty} \leqslant 1$. This implies

$$
\begin{aligned}
\sum_{j=0}^{J}\left|u_{j}^{1 / 2}\right| \leqslant & \lambda\left|g_{1 / 2}^{0}-\lambda q(0) \phi_{0}\right|+\mu d_{1 / 2}^{0}+\lambda\left|g_{J-1 / 2}^{0}-\Psi(0)\right|+\mu d_{J-1 / 2}^{0} \\
& +\sum_{j=0}^{J-1}\left|g_{j+1 / 2}^{0}-g_{j-1 / 2}^{0}-\mu\left(d_{j+1 / 2}^{0}-d_{j-1 / 2}^{0}\right)\right| .
\end{aligned}
$$

The last right-hand side is uniformly bounded due to (H4) and (H5).

4.2. Global estimates on $A\left(\phi_{\Delta}\right)$. In this section we derive a space and time translate estimate for $A\left(\phi_{\Delta}\right)$, where $\phi_{\Delta}$ is the linearly interpolated approximate solution of (1.1). The proof of the following Lemma 4.3 is a slight modification of a proof given in [22, which includes the boundary conditions, while the proof of Lemma 4.4 does to not appeal to boundary conditions, and is therefore a subcase of the derivation leading to Theorem 4.1 in 22 .

Lemma 4.3. If the CFL condition (2.7) holds, then there exists a constant $C_{6}$ independent of $\Delta$ such that

$$
\left|A\left(\phi_{j}^{n}\right)-A\left(\phi_{i}^{n}\right)\right| \leqslant C_{6}|j-i| \Delta x \quad \text { for } 0 \leqslant i, j \leqslant J .
$$

Proof. We choose $j \in\{0, \ldots, J-1\}$ and $i=j+1$ and estimate $\left|d_{j+1 / 2}^{n+1}\right|$. For $j=J-2$, the statement follows immediately from the following inequality, which is a consequence of the boundary scheme (2.6c):

$$
\frac{\left|d_{J-1 / 2}^{n+1}\right|}{\Delta x} \leqslant \frac{1}{\lambda}\left|\phi_{J}^{n+1}-\phi_{J}^{n}\right|+\left|g_{J-1 / 2}^{n}\right|+\|\Psi\|_{\infty} .
$$

For $j=0, \ldots, J-2$, we may use Lemma 4.2 to obtain

$$
\begin{aligned}
\frac{\left|d_{j+1 / 2}^{n+1}\right|}{\Delta x}-\left|g_{j+1 / 2}^{n}\right| & \leqslant\left|g_{j+1 / 2}^{n}-\frac{d_{j+1 / 2}^{n+1}}{\Delta x}\right| \\
& =\left|\sum_{m=1}^{j} \Delta_{+}\left(g_{m-1 / 2}^{n}-\frac{d_{m-1 / 2}^{n+1}}{\Delta x}\right)+g_{1 / 2}^{n}-\frac{d_{1 / 2}^{n+1}}{\Delta x}\right| \\
& \leqslant \frac{1}{\lambda} \sum_{m=0}^{j}\left|\phi_{m}^{n+1}-\phi_{m}^{n}\right|+\phi_{\max }\|q\|_{\infty} \leqslant C_{5}+\phi_{\max }\|q\|_{\infty}
\end{aligned}
$$

where we use the standard notation $\Delta_{+} V_{j}^{n}:=V_{j+1}^{n}-V_{j}^{n}, \Delta_{-} V_{j}^{n}:=V_{j}^{n}-V_{j-1}^{n}$. Inequalities (4.18) and (4.19) imply (4.17) for $n \geqslant 1$, while for $n=0$, (4.17) is ensured by assumption (H5). The statement of Lemma 4.3 follows with $C_{6}=$ $\max \left\{C_{5}+\phi_{\max }\|q\|_{\infty}+\|g\|_{\infty}, M_{0}+M_{1}\right\}$.

As a consequence of Lemma 4.3 , there exists a constant $C_{7}$ such that

$$
\lambda \sum_{j=0}^{J-1} \sum_{n=0}^{N-1}\left(A\left(\phi_{j+1}^{n}\right)-A\left(\phi_{j}^{n}\right)\right)^{2} \leq C_{7} .
$$

Lemma 4.4. Under the assumptions of Lemma 4.3, there exists a constant $C_{8}$ such that

$$
\left|A\left(\phi_{j}^{n}\right)-A\left(\phi_{j}^{m}\right)\right| \leqslant C_{8} \sqrt{|n-m| \Delta t}, \quad 0 \leqslant n, m \leqslant N, \quad j=0, \ldots, J .
$$


The proof of Lemma 4.4 which is omitted here, is analogous to the proof of Lemma 4.2 in [23], which in turn is an adaptation of a technique introduced in 19. It utilizes inequality (4.17) combined with an interpolation technique that is independent of the particular scheme being used.

In the sequel, we denote by $\phi_{\Delta}$ (where $\Delta=(\Delta x, \Delta t)$ ) the interpolant of degree one associated with the data points $\left\{\phi_{j}^{n}\right\}$; see [18, 19]. Note that $\phi_{\Delta}$ is continuous everywhere and differentiable almost everywhere. From Lemmas $3.2,4.1$ and 4.2 we deduce that there is a constant $C_{9}=C_{9}(T)$ such that

$$
\left\|\phi_{\Delta}\right\|_{L^{\infty}\left(Q_{T}\right)}+\mathrm{TV}_{Q_{T}}\left(\phi_{\Delta}\right) \leqslant C_{9}
$$

while Lemmas 4.3 and 4.4 imply that there is a constant $C_{10}$ such that

$$
\begin{aligned}
& \left|A\left(\phi_{\Delta}(y, \tau)\right)-A\left(\phi_{\Delta}(x, t)\right)\right| \\
& \quad \leqslant C_{10}(|x-y|+\sqrt{|t-\tau|}+\Delta x+\sqrt{\Delta t}) \quad \forall(x, t),(y, \tau) \in Q_{T} .
\end{aligned}
$$

\section{Convergence analysis}

In view of the embedding of $L^{\infty}\left(Q_{T}\right) \cap B V\left(Q_{T}\right)$ in $L^{1}\left(Q_{T}\right)$ [17, there exists a sequence $\left\{\Delta_{i}\right\}_{i \in \mathbb{N}}$ with $\Delta_{i} \rightarrow 0$ for $i \rightarrow \infty$ and a function $\phi \in L^{\infty}\left(Q_{T}\right) \cap B V\left(Q_{T}\right)$ such that $\phi_{\Delta} \rightarrow \phi$ a.e. on $Q_{T}$. Furthermore, the Arzelà-Ascoli theorem implies that $A\left(\phi_{\Delta}\right) \rightarrow A(\phi)$ uniformly on $Q_{T}$, and we have that $A(\phi) \in C^{1,1 / 2}\left(Q_{T}\right)$. It remains to show that $\phi$ satisfies the entropy condition (S3), and we need to examine the boundary conditions.

5.1. The discrete entropy inequality. We define the vectors $\mathbf{k}=(k, \ldots, k) \in$ $\mathbb{R}^{J+1}, \phi^{n} \top \mathbf{k}:=\left(\phi_{0}^{n} \top k, \ldots, \phi_{J}^{n} \top k\right)$, and $\phi^{n} \perp \mathbf{k}:=\left(\phi_{0}^{n} \perp k, \ldots, \phi_{J}^{n} \perp k\right)$.

Lemma 5.1. For the numerical solution $\left\{\phi_{j}^{n}\right\}$ of the IBVP (1.1) obtained by the scheme (2.6) under the CFL condition (2.7), the discrete entropy inequalities

$$
\begin{aligned}
& \left|\phi_{0}^{n+1}-k\right|-\left|\phi_{0}^{n}-k\right|+\lambda G_{1 / 2}^{n}-\mu \operatorname{sgn}\left(\phi_{0}^{n+1}-k\right)\left(A\left(\phi_{1}^{n+1}\right)-A\left(\phi_{0}^{n+1}\right)\right) \\
& +\lambda \operatorname{sgn}\left(\phi_{0}^{n+1}-k\right)\left(b(k)+q\left(t^{n}\right)\left(\phi_{0}^{n}-k\right)\right) \leq 0, \\
& \left|\phi_{j}^{n+1}-k\right|-\left|\phi_{j}^{n}-k\right|+\lambda\left(G_{j+1 / 2}^{n}-G_{j-1 / 2}^{n}\right)-\mu \operatorname{sgn}\left(\phi_{j}^{n+1}-k\right) \\
& \times\left(A\left(\phi_{j-1}^{n+1}\right)-2 A\left(\phi_{j}^{n+1}\right)+A\left(\phi_{j+1}^{n+1}\right)\right) \leq 0, \quad j=1, \ldots, J-1, \\
& \left|\phi_{J}^{n+1}-k\right|-\left|\phi_{J}^{n}-k\right|-\lambda G_{J-1 / 2}^{n}+\mu \operatorname{sgn}\left(\phi_{J}^{n+1}-k\right)\left(A\left(\phi_{J}^{n+1}\right)-A\left(\phi_{J-1}^{n+1}\right)\right) \\
& +\lambda \operatorname{sgn}\left(\phi_{J}^{n+1}-k\right)\left(\Psi\left(t^{n}\right)-q\left(t^{n}\right) k-b(k)\right) \leq 0
\end{aligned}
$$

hold for all $k \in \mathbb{R}$ with the discrete entropy flux

$$
G_{j+1 / 2}^{n}:=g\left(\phi_{j}^{n} \top k, \phi_{j+1}^{n} \top k, t^{n}\right)-g\left(\phi_{j}^{n} \perp k, \phi_{j+1}^{n} \perp k, t^{n}\right),
$$

where we use the standard notation $a \top b:=\max \{a, b\}$ and $a \perp b:=\min \{a, b\}$.

Proof. Let us consider the following explicit scheme obtained from (2.6) for $A \equiv 0$ :

$$
\begin{aligned}
& \tilde{\phi}_{0}^{n+1}=\tilde{\mathcal{H}}_{0}\left(\phi^{n}, t^{n}\right):=\phi_{0}^{n}-\lambda g_{1 / 2}^{n}+\lambda q\left(t^{n}\right) \phi_{0}^{n}, \\
& \tilde{\phi}_{j}^{n+1}=\tilde{\mathcal{H}}_{j}\left(\phi^{n}, t^{n}\right):=\phi_{j}^{n}-\lambda\left(g_{j+1 / 2}^{n}-g_{j-1 / 2}^{n}\right), \quad j=1, \ldots, J-1, \\
& \tilde{\phi}_{J}^{n+1}=\tilde{\mathcal{H}}_{J}\left(\phi^{n}, t^{n}\right):=\phi_{J}^{n}+\lambda g_{J-1 / 2}^{n}-\lambda \Psi\left(t^{n}\right) .
\end{aligned}
$$


Clearly, the explicit scheme (3.11) obtained from (2.6) for $A \equiv 0$ is monotone. We define $\tilde{\phi}_{j}^{n+1}:=\tilde{\mathcal{H}}_{j}\left(\phi^{n}, t^{n}\right)$ for $j=0, \ldots, J$, and note that

$$
\begin{aligned}
\tilde{k}_{0}^{n+1} & :=\tilde{\mathcal{H}}_{0}(\mathbf{k}, t)=k-\lambda b(k), \quad \tilde{k}_{J}^{n+1}:=\tilde{\mathcal{H}}_{J}(\mathbf{k}, t)=k+\lambda(b(k)+q(t) k-\Psi(t)), \\
\tilde{k}_{j}^{n+1} & :=\tilde{\mathcal{H}}_{j}(\mathbf{k}, t)=k, \quad j=1, \ldots, J-1 .
\end{aligned}
$$

Consider first the interior scheme. For $j=1, \ldots, J-1$, the identity $|a-b|=a \top b-$ $a \perp b$ and the monotonicity of $\tilde{\mathcal{H}}_{j}$, which implies $\tilde{\mathcal{H}}_{j}\left(\mathbf{x} \top \mathbf{y}, t^{n}\right) \geqslant \tilde{\mathcal{H}}_{j}\left(\mathbf{x}, t^{n}\right) \top \tilde{\mathcal{H}}_{j}\left(\mathbf{y}, t^{n}\right)$ and $\tilde{\mathcal{H}}_{j}\left(\mathbf{x} \perp \mathbf{y}, t^{n}\right) \leqslant \tilde{\mathcal{H}}_{j}\left(\mathbf{x}, t^{n}\right) \perp \tilde{\mathcal{H}}_{j}\left(\mathbf{y}, t^{n}\right)$, lead to

$$
\begin{aligned}
\left|\tilde{\phi}_{j}^{n+1}-\tilde{k}_{j}^{n+1}\right| & =\left|\tilde{\mathcal{H}}_{j}\left(\phi^{n}, t^{n}\right)-\tilde{\mathcal{H}}_{j}\left(\mathbf{k}, t^{n}\right)\right| \\
& =\tilde{\mathcal{H}}_{j}\left(\phi^{n}, t^{n}\right) \top \tilde{\mathcal{H}}_{j}\left(\mathbf{k}, t^{n}\right)-\tilde{\mathcal{H}}_{j}\left(\phi^{n}, t^{n}\right) \perp \tilde{\mathcal{H}}_{j}\left(\mathbf{k}, t^{n}\right) \\
& \leqslant \tilde{\mathcal{H}}_{j}\left(\phi^{n} \top \mathbf{k}, t^{n}\right)-\tilde{\mathcal{H}}_{j}\left(\phi^{n} \perp \mathbf{k}, t^{n}\right) \\
& =\left|\phi_{j}^{n}-k\right|-\lambda\left(G_{j+1 / 2}^{n}-G_{j-1 / 2}^{n}\right)
\end{aligned}
$$

for $j=1, \ldots, J-1$. On the other hand, we know that

$$
\tilde{\phi}_{j}^{n+1}=\phi_{j}^{n+1}-\mu\left(A\left(\phi_{j-1}^{n+1}\right)-2 A\left(\phi_{j}^{n+1}\right)+A\left(\phi_{j+1}^{n+1}\right)\right), \quad j=1, \ldots, J-1 .
$$

Consequently, we obtain for $j=1, \ldots, J-1$

$$
\begin{aligned}
\left|\tilde{\phi}_{j}^{n+1}-\tilde{k}_{j}^{n+1}\right| & =\left|\tilde{\phi}_{j}^{n+1}-k\right| \\
& \geqslant \operatorname{sgn}\left(\phi_{j}^{n+1}-k\right)\left[\phi_{j}^{n+1}-k-\mu\left(A\left(\phi_{j-1}^{n+1}\right)-2 A\left(\phi_{j}^{n+1}\right)+A\left(\phi_{j+1}^{n+1}\right)\right)\right] \\
& =\left|\phi_{j}^{n+1}-k\right|-\mu \operatorname{sgn}\left(\phi_{j}^{n+1}-k\right)\left(A\left(\phi_{j-1}^{n+1}\right)-2 A\left(\phi_{j}^{n+1}\right)+A\left(\phi_{j+1}^{n+1}\right)\right) .
\end{aligned}
$$

Combining this with (5.4), we obtain (5.2). For $j=0$, we obtain (instead of (5.4))

$$
\left|\tilde{\phi}_{0}^{n+1}-\tilde{k}_{0}^{n+1}\right| \leqslant\left|\phi_{0}^{n}-k\right|+\lambda q\left(t^{n}\right)\left|\phi_{0}^{n}-k\right|-\lambda G_{1 / 2}^{n} .
$$

On the other hand, we have

$$
\begin{aligned}
\left|\tilde{\phi}_{0}^{n+1}-\tilde{k}_{0}^{n+1}\right|= & \left|\tilde{\phi}_{0}^{n+1}-k+\lambda b(k)\right| \\
\geqslant & \left|\phi_{0}^{n+1}-k\right|-\mu \operatorname{sgn}\left(\phi_{0}^{n+1}-k\right)\left(A\left(\phi_{1}^{n+1}\right)-A\left(\phi_{0}^{n+1}\right)\right) \\
& +\lambda \operatorname{sgn}\left(\phi_{0}^{n+1}-k\right) b(k) .
\end{aligned}
$$

Combining (5.5) and (5.6), we get (5.1). For $j=J$ the analogous inequalities are

$$
\begin{aligned}
\left|\tilde{\phi}_{J}^{n+1}-\tilde{k}^{n+1}\right| \leqslant & \left|\phi_{J}^{n}-k\right|+\lambda G_{J-1 / 2}^{n}, \\
\left|\tilde{\phi}_{J}^{n+1}-\tilde{k}^{n+1}\right| \geqslant & \left|\phi_{J}^{n+1}-k\right|+\mu \operatorname{sgn}\left(\phi_{J}^{n+1}-k\right)\left(A\left(\phi_{J}^{n+1}\right)-A\left(\phi_{J-1}^{n+1}\right)\right) \\
& +\lambda \operatorname{sgn}\left(\phi_{J}^{n+1}-k\right)\left(\Psi\left(t^{n}\right)+q\left(t^{n}\right) k-b(k)\right),
\end{aligned}
$$

whose combination yields (5.3). 
5.2. Satisfaction of the entropy inequality. We now show that the limit function $\phi$ satisfies the entropy inequality (2.1).

Lemma 5.2. Let $\phi_{\Delta}$ be the linearly interpolated numerical solution of (1.1) and $\phi \in L^{\infty}\left(Q_{T}\right) \cap B V\left(Q_{T}\right)$ such that $\phi_{\Delta} \rightarrow \phi$ in $L_{\text {loc }}^{1}$. If $\phi_{\Delta}$ is obtained by the scheme (2.6) under the CFL condition (2.7), then $\phi$ satisfies the entropy inequality (2.1).

Proof. Let $\varphi \in C_{0}^{\infty}\left(Q_{T}\right)$ be a nonnegative function. We multiply the discrete entropy inequalities (5.1) -(5.3) by $\varphi\left(x, t^{n}\right)$, integrate the $j$-th inequality over the corresponding interval $I_{j}=\left[x_{j-1 / 2}, x_{j+1 / 2}\right)$, and sum over $(j, n) \in\{0, \ldots, J\} \times$ $\{0, \ldots, N-1\}$. This yields an inequality $E_{1}+\cdots+E_{4} \leq 0$, where

$$
\begin{aligned}
E_{1}:= & \sum_{n=0}^{N-1} \sum_{j=0}^{J}\left(\left|\phi_{j}^{n+1}-k\right|-\left|\phi_{j}^{n}-k\right|\right) \int_{I_{j}} \varphi\left(x, t^{n}\right) d x \\
E_{2}:= & \sum_{n=0}^{N-1} \sum_{j=1}^{J-1} \lambda\left(G_{j+1 / 2}^{n}-G_{j-1 / 2}^{n}\right) \int_{I_{j}} \varphi\left(x, t^{n}\right) d x \\
+ & \lambda \sum_{n=0}^{N-1}\left[G_{1 / 2}^{n} \int_{I_{0}} \varphi\left(x, t^{n}\right) d x-G_{J-1 / 2}^{n} \int_{I_{J}} \varphi\left(x, t^{n}\right) d x\right] \\
E_{3}:= & -\sum_{n=0}^{N-1} \sum_{j=1}^{J-1} \mu \operatorname{sgn}\left(\phi_{j}^{n+1}-k\right)\left[A\left(\phi_{j-1}^{n+1}\right)-2 A\left(\phi_{j}^{n+1}\right)+A\left(\phi_{j+1}^{n+1}\right)\right] \int_{I_{j}} \varphi\left(x, t^{n}\right) d x \\
+ & \mu \sum_{n=0}^{N-1}\left[\operatorname{sgn}\left(\phi_{J}^{n+1}-k\right)\left(A\left(\phi_{J}^{n+1}\right)-A\left(\phi_{J-1}^{n+1}\right)\right) \int_{I_{J}} \varphi\left(x, t^{n}\right) d x\right. \\
& \left.-\operatorname{sgn}\left(\phi_{0}^{n+1}-k\right)\left(A\left(\phi_{1}^{n+1}\right)-A\left(\phi_{0}^{n+1}\right)\right) \int_{I_{0}} \varphi\left(x, t^{n}\right) d x\right] \\
& \lambda \sum_{n=0}^{N-1}\left[\operatorname{sgn}\left(\phi_{0}^{n+1}-k\right)\left(b(k)+q\left(t^{n}\right)\left(k-\phi_{0}^{n}\right)\right) \int_{I_{0}} \varphi\left(x, t^{n}\right) d x\right. \\
& \left.-\operatorname{sgn}\left(\phi_{J}^{n+1}-k\right)\left(b(k)+q\left(t^{n}\right) k-\Psi\left(t^{n}\right)\right) \int_{I_{J}} \varphi\left(x, t^{n}\right) d x\right] .
\end{aligned}
$$

A summation by parts and using that $\varphi$ has compact support yield

$$
\begin{aligned}
E_{1}= & \sum_{j=0}^{J}\left[\left|\phi_{j}^{N}-k\right| \int_{I_{j}} \varphi(x, T) d x-\left|\phi_{j}^{0}-k\right| \int_{I_{j}} \varphi(x, 0) d x\right. \\
& \left.-\Delta t \sum_{n=0}^{N-2}\left|\phi_{j}^{n+1}-k\right| \int_{I_{j}} \frac{\varphi\left(x, t^{n+1}\right)-\varphi\left(x, t^{n}\right)}{\Delta t} d x\right] \\
= & -\Delta t \sum_{j=0}^{J} \sum_{n=0}^{N-2}\left|\phi_{j}^{n+1}-k\right| \int_{I_{j}} \frac{\varphi\left(x, t^{n+1}\right)-\varphi\left(x, t^{n}\right)}{\Delta t} d x .
\end{aligned}
$$


Another summation by parts, the consistency of $g$ with $f$, and Lemma 4.1 imply

$$
\begin{aligned}
E_{2} & =-\Delta t \sum_{n=0}^{N-1} \sum_{j=0}^{J-1} G_{j+1 / 2}^{n} \int_{I_{j}} \frac{\Delta_{+} \varphi\left(x, t^{n}\right)}{\Delta x} d x \\
& =-\Delta t \sum_{n=0}^{N-1} \sum_{j=0}^{J-1} \operatorname{sgn}\left(\phi_{j}^{n}-k\right)\left(f\left(\phi_{j}^{n}, t^{n}\right)-f\left(k, t^{n}\right)\right) \int_{I_{j}} \frac{\Delta_{+} \varphi\left(x, t^{n}\right)}{\Delta x} d x+\mathcal{O}(\Delta x),
\end{aligned}
$$

where $\Delta_{-} V\left(x, t^{n}\right):=V\left(x+\Delta x, t^{n}\right)-V\left(x, t^{n}\right)$. A summation by part shows that $E_{3}=E_{3}^{1}+E_{3}^{2}$, where

$$
\begin{aligned}
E_{3}^{1}:=\mu \sum_{n=0}^{N-1} \sum_{j=1}^{J}\left(\operatorname{sgn}\left(\phi_{j}^{n+1}-k\right)-\operatorname{sgn}\left(\phi_{j-1}^{n+1}-k\right)\right) \\
\quad \times\left(A\left(\phi_{j}^{n+1}\right)-A\left(\phi_{j-1}^{n+1}\right)\right) \int_{I_{j}} \varphi\left(x, t^{n}\right) d x, \\
E_{3}^{2}:=\lambda \sum_{n=0}^{N-1} \sum_{j=0}^{J-1} \operatorname{sgn}\left(\phi_{j}^{n+1}-k\right)\left(A\left(\phi_{j+1}^{n+1}\right)-A\left(\phi_{j}^{n+1}\right)\right) \int_{I_{j}} \frac{\Delta_{+} \varphi\left(x, t^{n}\right)}{\Delta x} d x .
\end{aligned}
$$

Noting that both $\operatorname{sgn}\left(\phi_{j}^{n+1}-k\right)-\operatorname{sgn}\left(\phi_{j-1}^{n+1}-k\right)$ and $A\left(\phi_{j}^{n+1}\right)-A\left(\phi_{j-1}^{n+1}\right)$ are both nonpositive or both nonnegative, we see that $E_{3}^{1} \geqslant 0$. On the other hand,

$$
\begin{aligned}
E_{3}^{2} & =\Delta t \sum_{n=0}^{N-1} \sum_{j=0}^{J-1} \operatorname{sgn}\left(\phi_{j}^{n}-k\right) \frac{A\left(\phi_{j+1}^{n}\right)-A\left(\phi_{j}^{n}\right)}{\Delta x} \int_{I_{j}} \frac{\Delta_{+} \varphi\left(x, t^{n}\right)}{\Delta x} d x+\mathcal{O}(\Delta x) \\
& =\iint_{Q_{T}} \operatorname{sgn}\left(\phi_{\Delta}-k\right) A\left(\phi_{\Delta}\right)_{x} \varphi_{x} d x d t+\mathcal{O}(\Delta x) .
\end{aligned}
$$

Since $\varphi$ and $\varphi_{x}$ vanish at $x=0$ and $x=1$, the integrals appearing in $E_{4}^{\Delta}$ are $\mathcal{O}\left(\Delta x^{2}\right)$ quantities, which implies $E_{4}=\mathcal{O}(\Delta x)$. Since $\varphi$ is smooth, we now may state the remaining inequality $-E_{1}-E_{2}-E_{3}^{1} \geqslant 0$ as

$$
\begin{gathered}
\iint_{Q_{T}}\left\{\left|\phi_{\Delta}-k\right| \varphi_{t}+\operatorname{sgn}\left(\phi_{\Delta}-k\right)\left[f\left(\phi_{\Delta}, t\right)-f(k, t)-A\left(\phi_{\Delta}\right)_{x}\right] \varphi_{x}\right\} d x d t \\
\geqslant-C_{11} \Delta x .
\end{gathered}
$$

Taking $\Delta \rightarrow 0$ and noting that $A\left(\phi_{\Delta}\right)_{x} \stackrel{\star}{\rightarrow} A(\phi)_{x}$ in $L^{\infty}\left(Q_{T}\right)$, we see that the limit function $\phi$ satisfies (2.1).

\subsection{Satisfaction of initial and boundary conditions.}

Lemma 5.3. Let $\phi_{\Delta}$ be the linearly interpolated numerical solution of the IBVP (1.1) and $\phi \in L^{\infty}\left(Q_{T}\right) \cap B V\left(Q_{T}\right)$ such that $\phi_{\Delta} \rightarrow \phi$ in $L^{1}$. If $\phi_{\Delta}$ is obtained by the scheme (2.6) under the CFL condition (2.7), then $\phi$ satisfies the boundary condition (S5) and the initial condition (S6).

Proof. Multiplying (2.6c) by $\int_{I_{J}} \varphi\left(x, t^{n}\right) d x$, where the test function $\varphi$ is given by $\varphi(x, t):=\Phi(t) \nu_{h}(x)$ with $\Phi \in C_{0}^{\infty}(\mathcal{T})$, summing the result over $n=0, \ldots, N-1$, 
using summation by parts and (2.6b), we get

$$
\begin{aligned}
& 0= \sum_{n=0}^{N-1}\left(\lambda \Psi\left(t^{n}\right)+\phi_{J}^{n+1}-\phi_{J}^{n}-\lambda g_{J-1 / 2}^{n}+\mu d_{J-1 / 2}^{n+1}\right) \Phi\left(t^{n}\right) \int_{I_{J}} \nu_{h}(x) d x \\
&= \Delta t \sum_{n=0}^{N-1} \sum_{j=0}^{J-1} \Psi\left(t^{n}\right) \Phi\left(t^{n}\right) \int_{I_{j}} \frac{\Delta_{+} \nu_{h}(x)}{\Delta x} d x+\sum_{n=0}^{N-1}\left(\phi_{J}^{n+1}-\phi_{J}^{n}\right) \Phi\left(t^{n}\right) \int_{I_{J}} \nu_{h}(x) d x \\
&-\Delta t \sum_{n=0}^{N-1}\left(g_{J-1 / 2}^{n}-\frac{d_{J-1 / 2}^{n+1}}{\Delta x}\right) \Phi\left(t^{n}\right) \int_{I_{J-1}} \frac{\Delta_{+} \nu_{h}(x)}{\Delta x} d x \\
&-\sum_{n=0}^{N-1} \sum_{j=0}^{J-2}\left[\left(\lambda g_{j+3 / 2}^{n}-\mu d_{j+3 / 2}^{n+1}\right) \int_{I_{j+1}} \nu_{h}(x) d x\right. \\
&\left.\quad \quad-\left(\lambda g_{j+1 / 2}^{n}-\mu d_{j+1 / 2}^{n+1}\right) \int_{I_{j}} \nu_{h}(x) d x\right] \Phi\left(t^{n}\right) \\
&=: \mathcal{S}_{1}+\mathcal{S}_{2}+\mathcal{S}_{3}+\mathcal{S}_{4} .
\end{aligned}
$$

First, we have that

$$
\mathcal{S}_{1}=\iint_{Q_{T}} \Psi(t) \Phi(t) \nu_{h}^{\prime}(x) d x+\mathcal{O}(\Delta x) .
$$

Assuming that $\Delta x$ is chosen small enough such that $\nu_{h}=0$ on $I_{0}$, we get

$$
\mathcal{S}_{2}=\left[\phi_{J}^{N} \Phi\left(t^{N-1}\right)-\sum_{n=1}^{N-1} \phi_{J}^{n}\left(\Phi\left(t^{n}\right)-\Phi\left(t^{n-1}\right)\right)\right] \int_{I_{J}} \nu_{h}(x) d x
$$

which implies

$$
\left|\mathcal{S}_{2}\right| \leqslant \phi_{\max }\left(\|\Phi\|_{\infty}+\operatorname{TV}_{\mathcal{T}}(\Phi)\right) \Delta x=C_{12} \Delta x .
$$

Moreover, we assume that $\Delta x<h$, which implies $\mathcal{S}_{3}=0$. Next, we get

$$
\begin{aligned}
\mathcal{S}_{4}=- & \sum_{n=0}^{N-1} \sum_{j=0}^{J-2}\left[\Delta_{+}\left(\lambda g_{j+1 / 2}^{n}-\mu d_{j+1 / 2}^{n+1}\right) \int_{I_{j+1}} \nu_{h}(x) d x\right. \\
& \left.+\left(\lambda g_{j+1 / 2}^{n}-\mu d_{j+1 / 2}^{n+1}\right) \int_{I_{j}} \Delta_{+} \nu_{h}(x) d x\right] \Phi\left(t^{n}\right) \\
=- & \sum_{n=0}^{N-1} \sum_{j=1}^{J-1}\left(\phi_{j}^{n+1}-\phi_{j}^{n}\right) \Phi\left(t^{n}\right) \int_{I_{j}} \nu_{h}(x) d x \\
& -\Delta t \sum_{n=0}^{N-1} \sum_{j=1}^{J-1}\left(g_{j-1 / 2}^{n}-\frac{d_{j-1 / 2}^{n+1}}{\Delta x}\right) \Phi\left(t^{n}\right) \int_{I_{j-1}} \frac{\Delta_{+} \nu_{h}(x)}{\Delta x} d x=: \mathcal{S}_{4}^{1}+\mathcal{S}_{4}^{2} .
\end{aligned}
$$

Note that

$$
\begin{aligned}
\mathcal{S}_{4}^{1} & =-\sum_{j=1}^{J-1}\left[\sum_{n=0}^{N-1}\left(\phi_{j}^{n+1}-\phi_{j}^{n}\right) \Phi\left(t^{n}\right)\right] \int_{I_{j}} \nu_{h}(x) d x \\
& =-\sum_{j=1}^{J-1}\left[\phi_{j}^{N} \Phi\left(t^{N-1}\right)+\sum_{n=1}^{N-1}\left(\Phi\left(t^{n}\right)-\Phi\left(t^{n-1}\right)\right) \phi_{j}^{n}\right] \int_{I_{j}} \nu_{h}(x) d x,
\end{aligned}
$$


which implies the estimate

$$
\left|\mathcal{S}_{4}^{1}\right| \leqslant \phi_{\max }\left(\|\Phi\|_{\infty}+\operatorname{TV}_{\mathcal{T}}(\Phi)\right) \int_{I} \nu_{h}(x) d x \leqslant C_{13} h,
$$

where the constant $C_{13}$ does not depend on $\Delta$ or $h$. Finally, we see that

$$
\begin{aligned}
\mathcal{S}_{4}^{2}= & -\Delta t \sum_{n=0}^{N-1} \sum_{j=1}^{J-1}\left(g_{j-1 / 2}^{n}-\frac{d_{j-1 / 2}^{n+1}}{\Delta x}\right) \Phi\left(t^{n}\right) \int_{I_{j-1}} \nu_{h}^{\prime}(x) d x+\mathcal{O}(\Delta x) \\
= & -\Delta t \sum_{n=0}^{N-1}\left[\sum_{j=1}^{J-1} g_{j-1 / 2}^{n} \Phi\left(t^{n}\right) \int_{I_{j-1}} \nu_{h}^{\prime}(x) d x+\frac{A\left(\phi_{0}^{n+1}\right)}{\Delta x} \int_{I_{0}} \nu_{h}^{\prime}(x) d x\right. \\
& \left.-\frac{A\left(\phi_{J-1}^{n+1}\right)}{\Delta x} \int_{I_{J-2}} \nu_{h}^{\prime}(x) d x+\sum_{j=1}^{J-2} A\left(\phi_{j}^{n+1}\right) \int_{I_{j-1}} \nu_{h}^{\prime \prime}(x) d x\right] \Phi\left(t^{n}\right)+\mathcal{O}(\Delta x) .
\end{aligned}
$$

We assume that $\Delta x$ is sufficiently small, such that $\nu_{h}^{\prime}=0$ on $I_{0}$ and $I_{J-2}$. This assumption implies

$$
\begin{aligned}
\mathcal{S}_{4}^{2}= & -\Delta t \sum_{n=0}^{N-1}\left[\sum_{j=1}^{J-1} g_{j-1 / 2}^{n} \int_{I_{j-1}} \nu_{h}^{\prime}(x) d x+\sum_{j=1}^{J-2} A\left(\phi_{j}^{n+1}\right) \int_{I_{j-1}} \nu_{h}^{\prime \prime}(x) d x\right] \Phi\left(t^{n}\right) \\
& +\mathcal{O}(\Delta x) \\
= & -\Delta t \sum_{n=0}^{N-1} \sum_{j=1}^{J-1} \int_{I_{j-1}}\left(g_{j-1 / 2}^{n} \nu_{h}^{\prime}(x)+A\left(\phi_{j}^{n+1}\right) \nu_{h}^{\prime \prime}(x)\right) \Phi\left(t^{n}\right) d x+\mathcal{O}\left(\Delta x\left(1+h^{-2}\right)\right) \\
= & -\iint_{Q_{T}}\left(f\left(\phi_{\Delta}, t\right) \nu_{h}^{\prime}(x)+A\left(\phi_{\Delta}\right) \nu_{h}^{\prime \prime}(x)\right) \Phi(t) d x d t+\mathcal{O}\left(\Delta x\left(1+h^{-2}\right)\right) .
\end{aligned}
$$

Using an integration by parts, we get

$$
\mathcal{S}_{4}^{2}=-\iint_{Q_{T}}\left(f\left(\phi_{\Delta}, t\right)-A\left(\phi_{\Delta}\right)_{x}\right) \Phi(t) \nu_{h}^{\prime}(x) d x d t+\mathcal{O}\left(\Delta x\left(1+h^{-2}\right)\right) .
$$

Combining (5.7), (5.9), $\mathcal{S}_{3}=0$, (5.10) and (5.11), we obtain

$$
\iint_{Q_{T}}\left(\Psi(t)-f\left(\phi_{\Delta}, t\right)+A\left(\phi_{\Delta}\right)_{x}\right) \Phi(t) \nu_{h}^{\prime}(x) d x d t=\mathcal{O}\left(\Delta x\left(1+h^{-2}\right)\right) .
$$

Jointly letting $\Delta \rightarrow 0$ and $h \rightarrow 0$ in such a way that $\Delta x / h^{2} \rightarrow 0$ (for example, by choosing $\Delta x=h^{3}$ ), and considering that $f\left(\phi_{\Delta}, t\right)-\partial_{x} A\left(\phi_{\Delta}\right)$ as well as its spatial total variation is uniformly bounded, we may pass to the limit in (5.12) to obtain

$$
\int_{0}^{T} \gamma_{1}\left(\Psi(t)-f\left(\phi_{\Delta}, t\right)+A\left(\phi_{\Delta}\right)_{x}\right) \Phi(t) d t=0,
$$

which implies that boundary condition (S5) is satisfied.

From Lemma 4.2 we deduce that

$$
\int_{I}\left|\phi_{\Delta}(x, \Delta t)-\phi_{\Delta}(x, 0)\right| d x \leqslant C \Delta t .
$$

Taking $\Delta t \rightarrow 0$ we see that the initial condition (S6) is satisfied.

For the general case $q \not \equiv 0$, we have not been able to prove that the limit $\phi$ satisfies (S4). The basic difficulty appears when one attempts to repeat the proof of Lemma 5.3 for the boundary $x=0$, starting from (2.6a), multiplying that scheme 
by $\int_{I_{0}} \varphi\left(x, t^{n}\right) d x$, where $\varphi(x, t)=\Phi(t) \mu_{h}(x)$, and summing the result over $n=$ $0, \ldots, N-1$. This procedure will lead to the necessity to estimate terms like

$$
\sum_{n=1}^{N} \sum_{j=1}^{J} q\left(t^{n}\right) \Phi\left(t^{n}\right)\left(\phi_{j+1}^{n}-\phi_{j}^{n}\right) \int_{I_{j}} \mu_{h}(x) d x .
$$

The latter would be possible if we had more accurate information on the behaviour of the discrete solution in an $\mathcal{O}(h)$ strip $[0, C \cdot h] \times \mathcal{T}$. Our present analysis does not exclude a strongly oscillatory (in time) boundary layer near $x=0$, although numerical experiments [4, 21 illustrate that this does not happen. A related question is whether the functions $\phi_{\Delta}(0, \cdot)$ converge to a meaningful function of $t$ as $\Delta \rightarrow 0$. However, for the practically important case of batch settling in a closed column $(q \equiv 0)$, we can straightforwardly prove that the boundary condition (S4) is satisfied by repeating the proof of Lemma 5.3 under the modifications given above.

The previous series of lemmas forms a proof of the following theorem.

Theorem 5.1. Assume that (H1)-(H6) hold. Under the CFL condition (2.7), the interpolated approximate solution $\phi_{\Delta}$ obtained by the semi-implicit scheme (2.6) converges in the strong topology of $L^{1}\left(Q_{T}\right)$ for $\Delta \rightarrow 0$ to a function $\phi \in L^{\infty}\left(Q_{T}\right) \cap$ $B V\left(Q_{T}\right)$, which has the properties (S1)-(S3), (S5) and (S6) stated in the definition of an entropy solution. In the special case $q \equiv 0$, the boundary condition at $x=0$, (S4), is also satisfied, and the limit function is an entropy solution.

\section{REFERENCES}

[1] S. Berres, R. Bürger, K.H. Karlsen, and E.M. Tory. Strongly degenerate parabolic-hyperbolic systems modeling polydisperse sedimentation with compression. SIAM J. Appl. Math., 64:4180, 2003. MR 2029124

[2] R. Bürger, S. Evje, and K.H. Karlsen. On strongly degenerate convection-diffusion problems modeling sedimentation-consolidation processes. J. Math. Anal. Appl., 247:517-556, 2000. MR.1769093 (2001d:35110)

[3] R. Bürger, S. Evje, K.H. Karlsen, and K.-A. Lie. Numerical methods for the simulation of the settling of flocculated suspensions. Chem. Eng. J., 80:91-104, 2000.

[4] R. Bürger and K.H. Karlsen. On some upwind schemes for the phenomenological sedimentation-consolidation model. J. Eng. Math., 41:145-166, 2001. MR.1866604(2002h:76090)

[5] R. Bürger and K.H. Karlsen. On a diffusively corrected kinematic-wave traffic model with changing road surface conditions. Math. Models Methods Appl. Sci., 13:1767-1799, 2003. MR2032211

[6] R. Bürger, K.H. Karlsen, N.H. Risebro, and J.D. Towers. Monotone difference approximations for the simulation of clarifier-thickener units. Comput. Visual. Sci., 6:83-91, 2004. MR2061269 (2005c:65070)

[7] R. Bürger, K.H. Karlsen, N.H. Risebro, and J.D. Towers. Well-posedness in $B V_{t}$ and convergence of a difference scheme for continuous sedimentation in ideal clarifier-thickener units. Numer. Math., 97:25-65, 2004. MR2045458 (2004m:35175)

[8] R. Bürger, K.H. Karlsen, and J.D. Towers. A model of continuous sedimentation of flocculated suspensions in clarifier-thickener units. SIAM J. Appl. Math., 65:882-940, 2005. MR2136036

[9] R. Bürger, W.L. Wendland, and F. Concha. Model equations for gravitational sedimentationconsolidation processes. Z. Angew. Math. Mech., 80:79-92, 2000. MR1742180(2000i:76121)

[10] J. Carrillo. Entropy solutions for nonlinear degenerate problems. Arch. Rat. Mech. Anal., 147:269-361, 1999. MR1709116 (2000m:35132)

[11] A. Coronel, F. James, and M. Sepúlveda. Numerical identification of parameters for a model of sedimentation processes. Inverse Problems, 19:951-972, 2003. MR:2005312 (2004h:35220)

[12] M.G. Crandall and A. Majda. Monotone difference approximations for scalar conservation laws. Math. Comp., 34:1-21, 1980. MR 0551288(81b:65079)

[13] M.G. Crandall and L. Tartar. Some relations between nonexpansive and order preserving mappings. Proc. Amer. Math. Soc., 78:385-390, 1980. MR0553381(81a:47054) 
[14] K. Deimling. Nonlinear Functional Analysis. Springer-Verlag, Berlin, 1985. MR0787404 (86j:47001)

[15] B. Engquist and S. Osher. One-sided difference approximations for nonlinear conservation laws. Math. Comp., 36:321-351, 1981. MR0606500 (82c:65056)

[16] M.S. Espedal and K.H. Karlsen. Numerical solution of reservoir flow models based on large time step operator splitting methods. In: M.S. Espedal, A. Fasano and A. Mikelić (Eds.), Filtration in Porous Media and Industrial Application, Lecture Notes in Mathematics, vol. 1734, Springer-Verlag, Berlin, 9-77, 2000. MR.1816143 (2002a:76115)

[17] L.C. Evans and R.C. Gariepy. Measure Theory and Fine Properties of Functions. CRC Press, Boca Raton, FL, 1992. MR1158660 (93f:28001)

[18] S. Evje and K.H. Karlsen. Degenerate convection-diffusion equations and implicit monotone difference schemes. In: M. Fey and R. Jeltsch (Eds.), Hyperbolic Problems: Theory, Numerics, Applications, Vol. I (Zürich, 1998), 285-294. Birkhäuser, Basel, 1999. MR1717198 (2000k:65137)

[19] S. Evje and K.H. Karlsen. Monotone difference approximations of $B V$ solutions to degenerate convection-diffusion equations. SIAM J. Numer. Anal., 37:1838-1860, 2000. MR1766850 (2001g:65110)

[20] R. Eymard, T. Gallouët, and R. Herbin. The finite volume method. In: P. Ciarlet and J.-L. Lions, editors, Handbook of Numerical Analysis, pp. 713-1020. North Holland, 2000. MR.1804748 (2002e:65138)

[21] P. Garrido, R. Burgos, F. Concha, and R. Bürger. Settling velocities of particulate systems: 13. Software for the batch and continuous sedimentation of flocculated suspensions. Int. J. Mineral Process., 73:131-144, 2004.

[22] K.H. Karlsen and N.H. Risebro. Convergence of finite difference schemes for viscous and inviscid conservation laws with rough coefficients. M2AN Math. Model. Numer. Anal. 35:239269, 2001. MR:1825698(2002b:35138)

[23] K.H. Karlsen, N.H. Risebro, and J.D. Towers. Upwind difference approximations for degenerate parabolic convection-diffusion equations with a discontinuous coefficient. IMA J. Numer. Anal., 22:623-664, 2002. MR1937244(2003i:65071)

[24] K.H. Karlsen, N.H. Risebro, and J.D. Towers. $L^{1}$ stability for entropy solutions of nonlinear degenerate parabolic convection-diffusion equations with discontinuous coefficients. Skr. K. Nor. Vid. Selsk., 49 pp. (2003). MR.2024741 (2004j:35149)

[25] S.N. Kružkov. First-order quasi-linear equations in several independent variables. Math. USSR Sb., 10:217-243, 1970. MR0267257(42:2159)

[26] P. Nelson. Traveling-wave solutions of the diffusively corrected kinematic-wave model. Math. Comp. Modelling, 35:561-579, 2002. MR1884018(2002k:90020)

[27] Z. Wu. A boundary value problem for quasilinear degenerate parabolic equations, MRC Technical Summary Report \#2484, University of Wisconsin, USA, 1983. MR0725168 (85j:35103)

[28] A.I. Vol'pert. The spaces $B V$ and quasilinear equations. Math. USSR Sb., 2:225-267, 1967. MR0216338 (35:7172)

[29] A.I. Vol'pert and S.I. Hudjaev. Cauchy's problem for degenerate second order quasilinear parabolic equations. Math. USSR Sb., 7:365-387, 1969. MR0264232 (41:8828)

[30] Z. Wu and J. Wang. Some results on quasilinear degenerate parabolic equations of second order. In: Proceedings of the 1980 Beijing Symposium on Differential Geometry and Differential Equations, Vols. 1, 2, 3 (Beijing, 1980), 1593-1609. Science Press, Beijing, 1982. MR0714393 (85f:35124)

Departamento de Ingeniería Matemática, Universidad de Concepción, Casilla 160-C, Concepción, Chile

E-mail address: rburger@ing-mat.udec.cl

Departamento de Ciencias Básicas, Facultad de Ciencias, Universidad del Bío-Bío, Casilla 447, Campus Fernando May, Chillán, Chile

E-mail address: acoronel@roble.fdo-may.ubiobio.cl

Departamento de Ingeniería Matemática, Universidad de Concepción, Casilla 160-C, Concepción, Chile

E-mail address: mauricio@ing-mat.udec.cl 